\title{
Apple Peel Biochemical Changes after Foliar Application of Combined Boron and Calcium II. Photosynthetic Pigments, Total Peroxides and Photochemical Efficiency
}

\author{
A. Mwije ${ }^{1,2}$, E. W. Hoffman ${ }^{2,3}$, E. Lötze ${ }^{2^{*}}$ \\ ${ }^{1}$ Makerere University, Kampala, Uganda \\ ${ }^{2}$ Stellenbosch University, Stellenbosch, South Africa \\ ${ }^{3}$ University of Queensland, Brisbane, Australia \\ Email: *elotze@sun.ac.za
}

How to cite this paper: Mwije, A., Hoffman, E.W. and Lötze, E. (2020) Apple Peel Biochemical Changes after Foliar Application of Combined Boron and Calcium II. Photosynthetic Pigments, Total Peroxides and Photochemical Efficiency. American Journal of Plant Sciences, 11, 939-964. https://doi.org/10.4236/ajps.2020.116068

Received: May 11, 2020

Accepted: June 27, 2020

Published: June 30, 2020

Copyright (c) 2020 by author(s) and Scientific Research Publishing Inc. This work is licensed under the Creative Commons Attribution International License (CC BY 4.0).

http://creativecommons.org/licenses/by/4.0/

\begin{abstract}
Understanding of the physiological effect of post-full-bloom foliar boron combined with calcium $(\mathrm{B}+\mathrm{Ca})$ on apple (Malus domestica) peel tissues is envisaged to give way to the unknown mode-of-action by which these mineral regimens suppress fruit sunburn-browning incidence in orchards. Promotion of this mineral approach among growers, as a certainly cheaper alternative to mitigate fruit sunburn-browning incidence in apple orchards necessitates clear elucidation of its mode-of-action. This study investigated peel photosynthetic pigments and total peroxides (as a measure of oxidative stress) in three apple cultivars, 'Cripps Pink', 'Golden Delicious' and 'Granny Smith' which were treated with four $\mathrm{B}+\mathrm{Ca}$ treatments varying in levels of $\mathrm{B}$ and $\mathrm{Ca}$ as well as inclusion of zinc $(\mathrm{Zn})$ in one treatment. Randomized complete block design experiments with five replications were conducted at commercial farms in Western Cape, South Africa. Significant $(p<0.05)$ treatment effect for major pigment aspects and total peroxides occurred in all cultivars, but with strong influence of cultivar and fruit age. For instance, effect of varying $\mathrm{B}, \mathrm{Ca}$ and possible $\mathrm{B}+\mathrm{Ca}$ duet-effect on photosynthetic pigments occurred in 'Cripps Pink', whereas the Zn-treatment was mainly responsible for significant treatment effects in both 'Golden Delicious' and 'Granny Smith' apples. Significant treatment effect for total peroxides occurred in 'Cripps Pink' and 'Granny Smith', yet significant interaction effect occurred with 'Golden Delicious', however, these significant results did not yield meaningful peel oxidative stress differences among the treatments. Foliar treatment differences in photochemical efficiency $(\mathrm{Fv} / \mathrm{Fm})$ were not sig-
\end{abstract}


nificant. The study concludes with firm evidence that foliar $\mathrm{B}+\mathrm{Ca}$ treatment composition has a significant effect on apple peel photosynthetic pigments depending on cultivar, and $\mathrm{Zn}$ is not desirable in the formulation of these treatments.

\section{Keywords}

Carotenoids, Chlorophyll, Total Peroxides, Malus domestica, 'Cripps Pink'

\section{Introduction}

Post-full-bloom foliar applications of boron combined with calcium $(\mathrm{B}+\mathrm{Ca})$ suppress fruit sunburn-browning incidence in apple orchards, however the underlying mode-of-action is not known [1] [2] [3] [4]. Without a clear explanation of such mode-of-action, it is difficult to promote this certainly cheaper approach in mitigating apple fruit sunburn-browning. The mode-of-action sought is highly envisaged to be a biochemical mechanism induced in apple fruit peel tissue by the foliar $\mathrm{B}+\mathrm{Ca}$ treatments [5] [6] [7]. Apple peel phenolic dynamics among foliar $\mathrm{B}+\mathrm{Ca}$ treatments did not yield clear treatment induced patterns [7], yet these treatments had significantly suppressed class 1 (very mild type) sunburn-browning in two distinct cultivars [3]. This raised need to investigate other apple peel biochemical aspects, particularly still those relevant to apple fruit sunburn-browning development, for instance, photosynthetic pigments [8] [9], and oxidative stress status and/or species [10] [11] [12].

Calcium $(\mathrm{Ca})$ is known to be a crucial co-factor in the reactions of the oxygen-evolving complex of photosystem (PS) II [13] [14], perhaps explaining the observed de novo repair of PS structures leading to photoprotection in groundnut (Arachis hypogaea) plants undergoing heat stress and treated with foliar Ca [15]. Increased chlorophyll was also reported by [16] after foliar Ca application in grasses undergoing heat stress. On the other hand, low quantities of boron (B) supplied to Mung bean plants (Vigna radiata) also increased chlorophyll and carotenoid levels with reduction in plant stress indicators [17]. In addition, foliar B in sweet cherry at full-bloom increased chlorophyll and carotenoid pigments [18]. These research findings raise possibility that foliar B+Ca may influence levels of apple peel chlorophylls as a physiological effect, and then suppress sunburn-browning if increments or preservations of chlorophyll pigments occur.

Additionally, $\mathrm{Ca}$ and $\mathrm{B}$ have key roles in plant cells, relating to structural and maintenance of membranes integrity [19] [20] [21], especially from oxidative-stress-driven lipid peroxidation phenomenon induced by metal toxicity, water deficiency, excess light and heat stresses among others [22] [23] [24] [25] [26], but [3], did not find significant cellular structural differences among these foliar $\mathrm{B}+\mathrm{Ca}$ treatments. Ordinarily, innate antioxidant mechanisms in plant tissues attenuate harmful effects of oxidative stress [27] [28] [29]. Notably, high 
light and temperature stresses induce oxidative stress in apple peel tissues which heralds sunburn-browning induction and severity development [10] [11] [12], and certainly, low apple peel oxidative stress level is as well important to realize low sunburn-browning incidence at fruit harvest [30] [31] [32] [33] [34]. Application of foliar $\mathrm{B}$ and/or $\mathrm{Ca}$ in plants including fruits reportedly promotes antioxidant capacity [27] [35] [36] [37] [38] [39], and this raises questions as to whether foliar $\mathrm{B}+\mathrm{Ca}$ treatments possibly reduce apple peel oxidative stress. Hence, in addition to photosynthetic pigment changes, this study investigated apple peel oxidative stress and photochemical efficiency ( $\mathrm{Fv} / \mathrm{Fm})$ of apple peel photosystem (PS) II in the three study cultivars treated with the varying foliar $\mathrm{B}+\mathrm{Ca}$ treatments.

\section{Materials and Methods}

\subsection{Experimental Setup and Treatments Design}

The study was conducted in the Western Cape, South Africa during the 2015/16 season using randomized complete block design experiment, with five replications for 'Cripps Pink', 'Golden Delicious' and 'Granny Smith'. These orchards

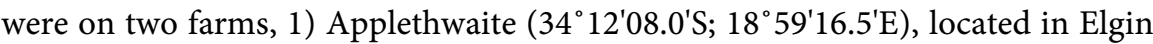
('Golden Delicious' and 'Granny Smith') and, 2) Welgevallen ( $33^{\circ} 56^{\prime} 52.5^{\prime} \mathrm{S}$; $18^{\circ} 52^{\prime} 19.9^{\prime} \mathrm{E}$ ) located in Stellenbosch ('Cripps Pink'). Single trees were used as experimental units, meaning that for the five experimental design replications, each replicate had five trees. All apple trees were trained as central leaders on M793 rootstock and they received fertilizers, pest and disease control, thinning and irrigation according to commercial practice.

The design of the foliar $\mathrm{B}+\mathrm{Ca}$ treatments followed indications and results obtained by [1] in regard to combining $B$ with $C$ a to suppress sunburn-browning in 'Golden Delicious' apples. The treatments were, 1) B'0.00+Ca'0.00 as control, 2) $\mathrm{B}^{\prime} 0.02+\mathrm{Ca}^{\prime} 0.06+\mathrm{Zn}^{\prime} 0.03$ (6 ml Manni-Plex ${ }^{\oplus} \mathrm{B}$ plus $10 \mathrm{ml}$ Manni-Plex ${ }^{\oplus}$ Cal-Zn), 3) B’0.02+Ca'1.24 (6 ml Manni-Plex ${ }^{\oplus}$ B plus $\left.65 \mathrm{~g} \mathrm{Calsol}^{\circledR}\right)$. Treatment (iv) $\mathrm{B}^{\prime} 0.08+\mathrm{Ca}^{\prime} 1.24\left(5 \mathrm{~g}^{2}\right.$ Spraybor ${ }^{\circledR}$ plus $\left.65 \mathrm{~g} \mathrm{Calsol}^{\circledR}\right)$ and treatment (v), B’0.17+Ca'1.24 (10 g Spraybor ${ }^{\circledast}$ with $\left.65 \mathrm{~g} \mathrm{Calsol}^{\circledR}\right)$. Treatment B+Ca nomenclature as used in this study represent respective $\mathrm{B}$ and Ca levels as weight/weight according to respective formulation product labels, as guided by composition of products indicated in Table 1 , for instance $\mathrm{B}$ 'X $+\mathrm{Ca}^{\prime} \mathrm{Y}$ infers $\mathrm{X} \mathrm{g} \cdot \mathrm{l}^{-1} \mathrm{~B}$ combined with $\mathrm{Y} \mathrm{g} \cdot \mathrm{l}^{-1} \mathrm{Ca}$ and then tank-mixed with $10 \mathrm{~L}$ of water. All the treatment formulation products were sourced from NexusAG (Pty) Ltd., South Africa, except Spraybor ${ }^{\circledast}$ which was got from Nulandis (Pty) Ltd., South Africa. The full-bloom in 'Cripps Pink' apples occurred on 5 October 2015, and the foliar application of the $\mathrm{B}+\mathrm{Ca}$ treatments began on 5 November 2015 (30 DAFB), and continued consecutively at weekly intervals on 12, 19, 27 November 2015 and 3, 10 December 2015. Full-bloom in both 'Golden Delicious' and 'Granny Smith' occurred on 16 October 2015, and the foliar treatments were applied starting at 25 November 2015 (39 DAFB), and as well continued consecutively at 
Table 1. Composition of the products used to formulate foliar treatments.

\begin{tabular}{cc}
\hline Trade name & Active Ingredients \\
\hline Manni-Plex Cal-Zn & $6 \% \mathrm{Ca}, 6 \% \mathrm{~N}, 3 \% \mathrm{Zn}$ \\
Manni-Plex ${ }^{\circ} \mathrm{B}$ & $3.3 \% \mathrm{~B}, 5 \% \mathrm{~N}$ \\
$\mathrm{Calsol}^{\circ}\left(5 \mathrm{Ca}\left(\mathrm{NO}_{3}\right)_{2} \mathrm{NH}_{4} \mathrm{NO}_{3} \cdot 10 \mathrm{H}_{2} \mathrm{O}\right)$ & $19 \% \mathrm{Ca}, 15.5 \% \mathrm{~N}$ \\
Spraybor $^{\circ}\left(\mathrm{Na}_{2} \mathrm{~B}_{4} \mathrm{O}_{7} \cdot 10 \mathrm{H}_{2} \mathrm{O}\right)$ & $16.5 \% \mathrm{~B}$ \\
\hline
\end{tabular}

weekly intervals on 2, 9, 15, 22 and 29 December 2015. In all the experiments, the foliar $\mathrm{B}+\mathrm{Ca}$ treatments were applied until run-off (high volume) using motorized Stihl ${ }^{\oplus}$ backpack sprayer and targeting the developing fruitlets.

\subsection{Quantitative Determination of Photosynthetic Pigments and Total Peroxides}

At selected fruit maturity stages/days after full bloom (DAFB), fruit was randomly picked by the row tree sides and within the outer tree canopy and later peeled in whole to obtain peels. Four fruit maturity stages towards harvest maturity were taken for each cultivar whereby for 'Cripps Pink', the fruit was sampled at 122,136, 150 and 164 DAFB, for 'Golden Delicious' at 80, 94, 108 and 122 DAFB, and in 'Granny Smith' fruit sampling was done at 94, 108, 122 and 136 DAFB. After fruit peeling, the peels were milled in liquid $\mathrm{N}$, and stored at $-80^{\circ} \mathrm{C}$ until quantitative determination of photosynthetic pigments and total peroxides.

The quantitative determination of apple peel photosynthetic pigments (chlorophylls and carotenoids) and total peroxides levels was conducted using analytical grade chemicals and solvents sourced from Sigma Aldrich, South Africa. These were, 1) Acetone 99.9\%, 2) Titanium Tetrachloride $\left.\left(\mathrm{TiCl}_{4}\right), 3\right)$ Hydrochloric acid $(37 \%)(\mathrm{HCl})$ and 4) Hydrogen Peroxide $\left(\mathrm{H}_{2} \mathrm{O}_{2}\right)$. Acetone stable plastic cuvettes (Lasec ${ }^{\oplus}$ (Pty) Ltd, South Africa) were used. The Milli-Q system (Millipore, Bedford, MA, USA), was the source of the double distilled water $\left(\mathrm{ddH}_{2} \mathrm{O}\right)$ as required. All centrifugation procedures at $3220 \mathrm{~g}$ and $20000 \mathrm{~g}$ were done at $4^{\circ} \mathrm{C}$ with Eppendorf ${ }^{\text {in }}$ 5810R and Eppendorf ${ }^{\text {in }}$ 5417R cooling centrifuges respectively, and a UV-visible light spectrophotometer (Cary 50 Bio, Varian, Australia (Pty) Ltd, Melbourne, Australia) was used for all absorbance measurements.

Chlorophyll pigments were determined following slightly modified method adopted from [40] [41]. Briefly, $500 \mathrm{mg}$ of fresh frozen apple peel was stirred with $3 \mathrm{ml}$ cold acetone in a $50 \mathrm{ml}$ capacity centrifuge tube at $4^{\circ} \mathrm{C}$ for 24 hours in darkness, followed by centrifugation for 15 minutes at $3220 \mathrm{~g}$, and extract was decanted into test tube and held at $4^{\circ} \mathrm{C}$ in darkness. Thereafter, an additional 2 $\mathrm{ml}$ cold acetone was added to the residues, followed by vortexing and centrifuging for 15 minutes at $3220 \mathrm{~g}$, and decanted as previously. The two-decants were combined, centrifuged for 15 minutes at $3220 \mathrm{~g}$, and $2 \mathrm{ml}$ of extract was pipetted off. The $2 \mathrm{ml}$ extract was centrifuged at $20000 \mathrm{~g}$ at $4^{\circ} \mathrm{C}$ for 15 minutes, and after $1 \mathrm{ml}$ was pipetted off and held at $4^{\circ} \mathrm{C}$ in darkness until determination of photo- 
synthetic pigments. Chlorophyll and carotenoid pigment levels were determined from absorbance values at 470, 645 and $670 \mathrm{~nm}$ following [40] [41], with acetone as the blank. Levels of chlorophyll $a$, chlorophyll $b$, total chlorophyll and total carotenoids were expressed as $\mu \mathrm{g} \cdot \mathrm{g}^{-1}$ apple peel fresh weight.

Total peroxides levels were determined with slightly modified protocols of [42] [43]. Briefly, $1 \mathrm{~g}$ of fresh frozen apple peel was added to $5 \mathrm{ml}$ of cold acetone, and homogenized using Ultra Turrax, IKA T18 basic $\left(14,000 \mathrm{~min}^{-1}\right)$ for 30 seconds, and homogenized mixture was centrifuged for 15 minutes at $4^{\circ} \mathrm{C}$ and $3220 \mathrm{~g}$. After, $2 \mathrm{ml}$ of supernatant was pipetted off and centrifuged at $4^{\circ} \mathrm{C}$ and $20000 \mathrm{~g}$ for 15 minutes. Then, $1 \mathrm{ml}$ of resulting supernatant was added to $12 \mathrm{ml}$ of $\mathrm{ddH}_{2} \mathrm{O}$, followed by vortexing. Thereafter, $1 \mathrm{ml}$ of Titanium reagent $(20 \%$ $\mathrm{TiCl}_{4}$ in $\mathrm{HCl}$ (37\%) volume per volume) was added, vortexed and held for $5 \mathrm{mi}$ nutes at room temperature, and then, $1 \mathrm{ml}$ of this mixture was used for absorbance measurement at $415 \mathrm{~nm}$ with $\mathrm{ddH}_{2} \mathrm{O}$ as the blank. The total peroxides levels were determined with standard curve made using freshly prepared commercial $\mathrm{H}_{2} \mathrm{O}_{2}$, ranging from 0.2 to $1.8 \mu \mathrm{M}$ and diluted from a stock of $2 \mu \mathrm{M}$ kept at $4^{\circ} \mathrm{C}$. The total peroxides levels were expressed as $\mu$ moles $(\mu \mathrm{mol})$ of $\mathrm{H}_{2} \mathrm{O}_{2}$ per gram of apple peel fresh weight.

\subsection{Evaluation of Apple Peel Photochemical Efficiency}

Immediately before respective fruit harvest of both 'Golden Delicious' and 'Granny Smith' apples, four fruit from both tree row-sides of the tree were randomly sampled, and placed in the dark, from where photochemical efficiency of photosystem II was measured as a ratio of variable to maximum chlorophyll fluorescence $(\mathrm{Fv} / \mathrm{Fm})$. The Fv/Fm values were recorded on both sun exposed and unexposed sides of fruit using a pulse modulated fluorimeter (FMS2, Hansatech Instruments Ltd., King's Lynn, Norfolk, England).

\subsection{Statistical Analyses}

The data was subjected to analysis of variance using Statistica software [44], and differences were deemed significant for $p<0.05$, where appropriate, means were separated with the Fisher's LSD posthoc test.

\section{Results}

\subsection{Photosynthetic Pigments and Total Peroxides}

\subsection{1. 'Cripps Pink' Apples}

There was no significant interaction $\left(\mathrm{B}+\mathrm{Ca}{ }^{\star} \mathrm{DAFB}\right)$ effect for any photosynthetic pigment aspect as well as total peroxides (Table 2), but foliar $\mathrm{B}+\mathrm{Ca}$ had a significant treatment effect except for chlorophyll $a$ to chlorophyll $b$ ratio and total carotenoids. Chlorophyll $a$, chlorophyll $b$, total chlorophyll and total chlorophyll to total carotenoids ratio patterns were largely similar among $\mathrm{B}^{\prime} 0.02+\mathrm{Ca}^{\prime} 1.24$, B'0.08+Ca'1.24 and B'0.17+Ca'1.24 where highest and insignificant differing values were recorded between them. However, only treatment B'0.17+Ca'1.24 
Table 2. 'Cripps Pink' apple peel photosynthetic pigments and total peroxides.

\begin{tabular}{|c|c|c|c|c|c|c|c|}
\hline Treatments $(\mathrm{B}+\mathrm{Ca})$ & $\mathrm{CHLa}^{1}$ & $\mathrm{CHLb}^{1}$ & $\mathrm{TCHL}^{1}$ & $\mathrm{TCAR}^{1}$ & CHLa:CHLb & TCHL:TCAR & TPERO $^{2}$ \\
\hline $\mathrm{B}^{\prime} 0.00+\mathrm{Ca}^{\prime} 0.00$ & $27.19^{\mathrm{bc}}$ & $18.12^{\mathrm{bc}}$ & $45.31^{\mathrm{bc}}$ & $12.21^{\mathrm{ns}}$ & $2.01^{\mathrm{ns}}$ & $3.77^{\mathrm{bc}}$ & $5.89^{\mathrm{a}}$ \\
\hline $\mathrm{B}^{\prime} 0.02+\mathrm{Ca}^{\prime} 1.24$ & $29.39^{\mathrm{a}}$ & $20.95^{\mathrm{ab}}$ & $50.33^{\mathrm{a}}$ & 12.63 & 1.53 & $4.08^{\mathrm{ab}}$ & $6.06^{\mathrm{a}}$ \\
\hline $\mathrm{B}^{\prime} 0.02+\mathrm{Ca}^{\prime} 0.06+\mathrm{Zn} \mathrm{n}^{\prime} 0.03$ & $26.09^{c}$ & $16.65^{c}$ & $42.74^{\mathrm{c}}$ & 12.86 & 1.86 & $3.38^{\mathrm{c}}$ & $5.87^{\mathrm{a}}$ \\
\hline B’0.08+Ca'1.24 & $28.25^{\mathrm{ab}}$ & $21.66^{\mathrm{a}}$ & $49.90^{\mathrm{ab}}$ & 11.29 & 1.53 & $4.60^{\mathrm{a}}$ & $5.32^{\mathrm{ab}}$ \\
\hline $\mathrm{B}^{\prime} 0.17+\mathrm{Ca}^{\prime} 1.24$ & $30.04^{\mathrm{a}}$ & $22.78^{\mathrm{a}}$ & $52.82^{\mathrm{a}}$ & 12.43 & 1.41 & $4.43^{\mathrm{a}}$ & $4.58^{\mathrm{b}}$ \\
\hline$p$ & 0.0019 & 0.0028 & 0.0007 & 0.0923 & 0.1305 & 0.0024 & 0.0033 \\
\hline \multicolumn{8}{|c|}{ Days after full bloom (DAFB) } \\
\hline 122 & $35.40^{\mathrm{a}}$ & $27.55^{\mathrm{a}}$ & $62.95^{\mathrm{a}}$ & $13.67^{\mathrm{a}}$ & $1.36^{\mathrm{b}}$ & $4.77^{\mathrm{a}}$ & $5.60^{\mathrm{b}}$ \\
\hline 136 & $29.45^{\mathrm{b}}$ & $24.26^{\mathrm{b}}$ & $53.72^{\mathrm{b}}$ & $11.33^{\mathrm{b}}$ & $1.38^{\mathrm{b}}$ & $4.98^{\mathrm{a}}$ & $5.71^{\mathrm{ab}}$ \\
\hline 150 & $23.89^{c}$ & $13.77^{\mathrm{c}}$ & $37.66^{c}$ & $12.52^{\mathrm{ab}}$ & $2.04^{\mathrm{a}}$ & $3.07^{\mathrm{b}}$ & $6.37^{\mathrm{a}}$ \\
\hline 164 & $24.01^{c}$ & $14.54^{c}$ & $38.55^{\mathrm{bc}}$ & $11.62^{\mathrm{b}}$ & $1.90^{\mathrm{a}}$ & $3.37^{\mathrm{b}}$ & $4.49^{c}$ \\
\hline$p$ & 0.0000 & 0.0000 & 0.0001 & 0.0001 & 0.0056 & 0.0000 & 0.0000 \\
\hline$p\left(\mathrm{~B}+\mathrm{Ca}^{\star} \mathrm{DAFB}\right)$ & 0.6232 & 0.4092 & 0.5722 & 0.2332 & 0.4819 & 0.2942 & 0.1780 \\
\hline
\end{tabular}

${ }^{1} \mu \mathrm{g} \cdot \mathrm{g}^{-1}$ apple peel fresh weight, ${ }^{2} \mathrm{nmol} \cdot \mathrm{g}^{-1}$ apple peel fresh weight, $\mathrm{CHLa}=$ Chlorophyll $\mathrm{a}$, CHLb $=$ Chlorophyll $b$, TCHL $=$ Total Chlorophyll, CHLa:CHLb $=$ Chlorophyll $a$ to Chlorophyll $b$ ratio, TCAR $=$ Total Carotenoids, TCHL:TCAR $=$ Total Chlorophyll to Total Carotenoids ratio and TPERO $=$ Total Peroxides.

showed consistent significant differences in chlorophylls and total chlorophyll to total carotenoids ratio from the control. But, B'0.08+Ca'1.24 was only significantly different from the control with only chlorophyll $b$ and total chlorophyll to total carotenoids ratio, while $\mathrm{B}^{\prime} 0.02+\mathrm{Ca}^{\prime} 1.24$ did significantly differ with control for only chlorophyll $a$ and total chlorophyll. Treatment B'0.02+Ca'0.06+Zn'0.03 had lowest levels of chlorophyll $a$, chlorophyll $b$, total chlorophyll and total chlorophyll to total carotenoids ratio that were not significantly different from the control, but differing with all other treatment, yielding a sharp contrast between this zinc $(\mathrm{Zn})$ containing treatment against all other $\mathrm{B}+\mathrm{Ca}$ treatments. In addition, treatment effect on total peroxides was significant, and treatment B'0.17+Ca'1.24 had lowest and statistically different value from the control and all other treatments, except B'0.08+Ca'1.24. The 'Cripps Pink' apple peel total peroxides levels associated with the control, B'0.02+Ca'1.24, B'0.02+Ca'0.06+Zn'0.03 and $\mathrm{B}^{\prime} 0.08+\mathrm{Ca}^{\prime} 1.24$ did not differ significantly.

The effect of fruit maturity stages (DAFB) was significant on all 'Cripps Pink' apple peel photosynthetic pigments aspects as well as total peroxides (Table 2). Values of chlorophyll $a$, chlorophyll $b$ and total chlorophyll significantly declined with progress of DAFB, with levels recorded at 122 DAFB significantly higher compared to all other DAFB periods. At 136 DAFB, chlorophyll a, chlorophyll $b$ and total chlorophyll were significantly higher from the indifferent 150 and 164 DAFB values, but total chlorophyll at 136 and 164 DAFB was as well not 
different. The total carotenoids at 122 DAFB was significantly different from all others with exception of that recorded at 150 DAFB. However, total carotenoids values at 136, 150 and 164 DAFB periods did not differ significantly, but generally total carotenoids decreased with advance of DAFB. The total chlorophyll to total carotenoids ratio at 136 and 122 DAFB was not significantly different, but these two values were all significantly different from the indifferent total chlorophyll to total carotenoids ratio values of both 150 and 164 DAFB, thus showing significant decrease from 136 to 150 DAFB. In contrast, chlorophyll a to chlorophyll $b$ ratio increased with DAFB progress, 122 and 136 DAFB had the lowest and not significantly differing values which jointly differed significantly with the higher indifferent values recorded at both 150 and 164 DAFB, the significant increase occurred from 136 to 150 DAFB. In addition, total peroxides also significantly increased, but only from 122 to 150 DAFB (Table 2). The lowest total peroxides level was at $164 \mathrm{DAFB}$, which was significantly lower from values at all other DAFB periods.

\subsection{2. 'Golden Delicious' Apples}

There was no significant interaction $\left(\mathrm{B}+\mathrm{Ca}^{*} \mathrm{DAFB}\right)$ effect for all photosynthetic pigment aspects (Table 3 ). Foliar $\mathrm{B}+\mathrm{Ca}$ treatment effect was significant treatment effects occurred for only chlorophyll $a$, total chlorophyll and total carotenoids aspects, where levels of these three aspects had similar variability patterns, characterized by treatment B'0.02+Ca'0.06+Zn'0.03 having lowest levels significantly different from values associated with all treatments and control, except chlorophyll $a$ and total chlorophyll values of B'0.08+Ca'1.24. The chlorophyll $a$, total chlorophyll and total carotenoids values of the control, B'0.02+Ca'1.24, B'0.08+Ca'1.24 and B'0.17+Ca'1.24 did not differ significantly. As was observed with 'Cripps Pink', a general sharp contrast in chlorophyll a, total chlorophyll and total carotenoids occurred between B'0.02+Ca'0.06+Zn'0.03 and all other treatments, except that in 'Golden Delicious', this $\mathrm{Zn}$ containing treatment had insignificant chlorophyll $a$ and total chlorophyll differences with B'0.08+Ca'1.24.

Days after full bloom (DAFB) had significant effect on all photosynthetic pigment attributes, and chlorophyll $a$, chlorophyll $b$, total chlorophyll and total carotenoids showed similar patterns as fruit maturity progressed (Table 3), where significantly differing highest and lowest values of these four aspects were recorded at 80 and 122 DAFB respectively, showing significant decrease as 'Golden Delicious' fruit maturated. Generally, chlorophyll $a$ to chlorophyll $b$ ratio decreased from 80 to $108 \mathrm{DAFB}$, and rose by $122 \mathrm{DAFB}$ to indifferent levels with both 80 and 94 DAFB periods. As was with 'Cripps Pink', total chlorophyll to total carotenoids ratio pattern among DAFB periods in 'Golden Delicious' was an exact reverse of the chlorophyll a:chlorophyll $b$. Thus, total chlorophyll to total carotenoids ratio generally increased from 80 to 108 , before decreasing at 122 DAFB to levels indifferent from those at both 80 and 108 DAFB periods. 
Table 3. 'Golden Delicious' apple peel photosynthetic pigments.

\begin{tabular}{ccccccc}
\hline Source & CHLa $^{1}$ & CHLb $^{1}$ & TCHL $^{1}$ & TCAR $^{1}$ & CHLa:CHLb & TCHL:TCAR \\
\hline Treatments (B+Ca) & & & & & & \\
B'0.00+Ca'0.00 & $55.25^{\mathrm{a}}$ & $39.14^{\mathrm{ns}}$ & $94.39^{\mathrm{a}}$ & $28.95^{\mathrm{a}}$ & $1.41^{\mathrm{ns}}$ & $3.27^{\mathrm{ns}}$ \\
B'0.02+Ca'1.24 & $54.85^{\mathrm{a}}$ & 39.43 & $94.27^{\mathrm{a}}$ & $27.37^{\mathrm{a}}$ & 1.40 & 3.45 \\
B'$^{\prime} 02+\mathrm{Ca}^{\prime} 0.06+\mathrm{Zn}^{\prime} 0.03$ & $48.18^{\mathrm{b}}$ & 35.64 & $83.82^{\mathrm{b}}$ & $24.36^{\mathrm{b}}$ & 1.36 & 3.47 \\
B'0.08+Ca'1.24 & $52.12^{\mathrm{ab}}$ & 38.21 & $90.33^{\mathrm{ab}}$ & $27.91^{\mathrm{a}}$ & 1.36 & 3.26 \\
B'$^{\prime} 0.17+\mathrm{Ca}^{\prime} 1.24$ & $55.10^{\mathrm{a}}$ & 40.06 & $95.16^{\mathrm{a}}$ & $28.45^{\mathrm{a}}$ & 1.38 & 3.37 \\
$p$ & 0.0056 & 0.1079 & 0.0218 & 0.0011 & 0.0529 & 0.1899 \\
Days after full bloom & & & & & & \\
$(\mathrm{DAFB})$ & & & & & & $3.37^{\mathrm{b}}$ \\
80 & $65.18^{\mathrm{a}}$ & $46.39^{\mathrm{a}}$ & $111.57^{\mathrm{a}}$ & $33.22^{\mathrm{a}}$ & $1.41^{\mathrm{a}}$ & $3.33^{\mathrm{b}}$ \\
94 & $52.41^{\mathrm{b}}$ & $37.90^{\mathrm{b}}$ & $90.30^{\mathrm{b}}$ & $27.35^{\mathrm{b}}$ & $1.39^{\mathrm{a}}$ & $3.57^{\mathrm{a}}$ \\
108 & $49.90^{\mathrm{b}}$ & $37.16^{\mathrm{b}}$ & $87.06^{\mathrm{b}}$ & $24.48^{\mathrm{c}}$ & $1.35^{\mathrm{b}}$ & $3.18^{\mathrm{b}}$ \\
122 & $44.92^{\mathrm{c}}$ & $32.53^{\mathrm{c}}$ & $77.45^{\mathrm{c}}$ & $24.59^{\mathrm{c}}$ & $1.39^{\mathrm{a}}$ & 0.0029 \\
$p$ & 0.0000 & 0.0000 & 0.0000 & 0.0000 & 0.0158 & 0.5475 \\
\hline (B+Ca $\left.{ }^{*} \mathrm{DAFB}\right)$ & 0.1024 & 0.2245 & 0.1481 & 0.0866 & 0.1251 & \\
\hline
\end{tabular}

${ }^{1} \mu \mathrm{g} \cdot \mathrm{g}^{-1}$ apple peel fresh weight, $\mathrm{CHLa}=$ Chlorophyll $a, \mathrm{CHLb}=$ Chlorophyll $b, \mathrm{TCHL}=$ Total Chlorophyll, CHLa:CHLb $=$ Chlorophyll $a$ to Chlorophyll $b$ ratio, TCAR $=$ Total Carotenoids and TCHL:TCAR $=$ Total Chlorophyll to Total Carotenoids ratio.

A significant interaction (B+Ca*DAFB) effect occurred for 'Golden Delicious' apple peel total peroxides (Table 4). At $80 \mathrm{DAFB}$, all treatments did not differ significantly, whereas at $94 \mathrm{DAFB}$ the control's highest total peroxides differed significantly with only values of B'0.02+Ca' 1.24 and B'0.02+Ca'0.06+Zn'0.03. At $94 \mathrm{DAFB}$, all foliar $\mathrm{B}+\mathrm{Ca}$ treatments did significantly differ from each other. At $108 \mathrm{DAFB}$, total peroxides of the control did not significantly differ with any foliar $\mathrm{B}+\mathrm{Ca}$ treatment. Significant total peroxides differences at 108 DAFB occurred only with B'0.08+Ca'1.24 (higher) and B'0.02+Ca'0.06+Zn'0.03 (lower). At $122 \mathrm{DAFB}$, total peroxides value of the control was only significantly different to that of B'0.08+Ca'1.24, and among treatments significant differences were only between the $\mathrm{Zn}$ treatment (highest) and both B'0.02+Ca'1.24 and B'0.08+Ca'1.24.

Generally, apple peel total peroxides levels decreased with fruit maturity, as values recorded at $80 \mathrm{DAFB}$ were significantly higher than those at $122 \mathrm{DAFB}$ (Table 4), except in control and B'0.02+Ca'0.06+Zn'0.03. Only treatment B'0.02+Ca'0.06+Zn'0.03 showed non-significantly differing total peroxides levels across all fruit maturity stages. Furthermore, treatments B'0.08+Ca'1.24 and $\mathrm{B}^{\prime} 0.17+\mathrm{Ca}^{\prime} 1.24$ showed non-significantly differing total peroxides levels from 80 to $108 \mathrm{DAFB}$, and the same was with treatment $\mathrm{B}^{\prime} 0.17+\mathrm{Ca}^{\prime} 1.24$ from 94 to 122 DAFB. At 108 and 122 DAFB, only treatments B'0.08+Ca'1.24 and B'0.02+Ca'1.24 showed significant differences in total peroxides levels between the two advanced fruit maturity stages. Generally, the interaction $\left(\mathrm{B}+\mathrm{Ca}^{\star} \mathrm{DAFB}\right)$ effect analysis (Table 4) did not reveal any foliar $\mathrm{B}+\mathrm{Ca}$ treatment 
Table 4. 'Golden Delicious' apple peel total peroxides'.

\begin{tabular}{ccccc}
\hline Treatments (B+Ca) & \multicolumn{4}{c}{ Days after full bloom (DAFB) } \\
\hline B'0.00+Ca'0.00 & 80 & 94 & 108 & 122 \\
\cline { 2 - 4 } B'0.02+Ca'1.24 & $9.4^{\mathrm{abcd}}$ & $10.7^{\mathrm{a}}$ & $8.3^{\mathrm{bcd}}$ & $7.7^{\text {cde }}$ \\
B'0.02+Ca'0.06+Zn'0.03 & $10.4^{\mathrm{ab}}$ & $7.6^{\text {cde }}$ & $8.9^{\mathrm{abcd}}$ & $5.6^{\text {ef }}$ \\
B'0.08+Ca'1.24 & $9.4^{\mathrm{abcd}}$ & $7.6^{\text {cde }}$ & $7.4^{\text {cdef }}$ & $8.3^{\mathrm{bcd}}$ \\
B'0.17+Ca'1.24 & $9.6^{\mathrm{abc}}$ & $9.4^{\mathrm{abcd}}$ & $10.1^{\mathrm{ab}}$ & $5.1^{\mathrm{f}}$ \\
\hline
\end{tabular}

${ }^{1} \mathrm{nmol} \cdot \mathrm{g}^{-1}$ peel fresh weight, $p\left(\mathrm{~B}+\mathrm{Ca}^{\star} \mathrm{DAFB}\right)=0.0276$.

that showed clearly distinguishable increasing or decreasing total peroxides patterns as 'Golden Delicious' fruit maturated from 80 to 122 DAFB.

\subsection{3. 'Granny Smith' Apples}

In 'Granny Smith' apples, a significant interaction $\left(B+\mathrm{Ca}^{*} \mathrm{DAFB}\right)$ effect occurred for all photosynthetic pigment aspects (Table 5), with exception of the ratios and total peroxides (Table 6). No significant differences were recorded for chlorophyll $a$, chlorophyll $b$, total chlorophyll and total carotenoids levels at 94 DAFB, except treatment B'0.02+Ca'1.24 whose lower values significantly differed with higher values obtained with B'0.08+Ca'1.24. At 108 DAFB, treatments and control did as well not differ significantly from each other for chlorophyll $a$, chlorophyll $b$, and total chlorophyll, except treatment B'0.02+Ca'0.06+Zn'0.03 with lowest values that significantly differed with all other values at this DAFB period. The total carotenoids levels at $108 \mathrm{DAFB}$ was also not significantly different between treatments and control, except the lowest value of associated with B'0.02+Ca'0.06+Zn'0.03 which significantly differed with the control and all treatments save B'0.17+Ca'1.24. At $122 \mathrm{DAFB}$, there was no foliar $\mathrm{B}+\mathrm{Ca}$ treatment difference with the control for chlorophyll $a$, chlorophyll $b$, total chlorophyll and total carotenoids that were significant. Yet, among treatments, only the highest values of $\mathrm{B}^{\prime} 0.02+\mathrm{Ca}^{\prime} 1.24$ were significantly different with values recorded with B'0.02+Ca'0.06+Zn'0.03 (except total carotenoids) and B'0.08+Ca'1.24. Also, at $136 \mathrm{DAFB}$, no treatment had significant differences from the control, except the lowest values of treatment $\mathrm{B}^{\prime} 0.02+\mathrm{Ca}^{\prime} 0.06+\mathrm{Zn}^{\prime} 0.03$, which were as well significantly different from all other treatments except B'0.08+Ca'1.24.

As 'Granny Smith' apples maturated, the control and B'0.17+Ca'1.24 consistently showed no significant chlorophyll $a$, chlorophyll $b$, total chlorophyll and total carotenoids differences from 94 to 136 DAFB. From 94 to 108 DAFB, treatment B'0.08+Ca'1.24 showed higher levels of chlorophyll $b$, total chlorophyll and total carotenoids which were jointly different from values recorded at 122 and $136 \mathrm{DAFB}$, thus only this treatment had chlorophyll $b$, total chlorophyll and total carotenoids as well as chlorophyll a clearly decreasing as fruit maturated. Overall, across 94 to $136 \mathrm{DAFB}$, chlorophyll $a$, chlorophyll $b$, total chlorophyll and total carotenoids levels were generally and significantly lowest in treatment $\mathrm{B}^{\prime} 0.02+\mathrm{Ca}^{\prime} 0.06+\mathrm{Zn}^{\prime} 0.03$, yet all other treatments did not differ amongst themselves and with the control. 
Table 5. 'Granny Smith’ peel photosynthetic pigments.

\begin{tabular}{|c|c|c|c|c|c|}
\hline Pigments $^{1}$ & Treatments $(\mathrm{B}+\mathrm{Ca})$ & \multicolumn{4}{|c|}{ Days after full bloom (DAFB) } \\
\hline \multirow{6}{*}{ Chlorophyll $a^{2}$} & & 94 & 108 & 122 & 136 \\
\hline & $\mathrm{B}^{\prime} 0.00+\mathrm{Ca}^{\prime} 0.00$ & $161.06^{\mathrm{abc}}$ & $154.71^{\mathrm{abcd}}$ & $153.33^{\mathrm{abcd}}$ & $145.58^{\text {cde }}$ \\
\hline & B’0.02+Ca'1.24 & $144.21^{\text {cde }}$ & $159.32^{\mathrm{abcd}}$ & $172.36^{\mathrm{a}}$ & $138.48^{\text {def }}$ \\
\hline & B'0.02+Ca'0.06+Zn'0.03 & $155.26^{\mathrm{abcd}}$ & $119.74^{\mathrm{fg}}$ & $145.59^{\text {cde }}$ & $113.37^{\mathrm{g}}$ \\
\hline & B'0.08+Ca'1.24 & $168.45^{\mathrm{ab}}$ & $157.80^{\mathrm{abcd}}$ & $139.21^{\mathrm{def}}$ & $128.63^{\text {efg }}$ \\
\hline & B'0.17+Ca'1.24 & $151.87^{\mathrm{abcd}}$ & $146.86^{\mathrm{cde}}$ & $158.26^{\mathrm{abcd}}$ & $150.68^{\mathrm{bcd}}$ \\
\hline \multirow{5}{*}{ Chlorophyll $b^{3}$} & $\mathrm{~B}^{\prime} 0.00+\mathrm{Ca}^{\prime} 0.00$ & $121.96^{\mathrm{abcd}}$ & $124.53^{\mathrm{abc}}$ & $119.17^{\mathrm{abcd}}$ & $112.86^{\text {bcde }}$ \\
\hline & B'0.02+Ca'1.24 & $108.71^{\text {cdef }}$ & $126.68^{\mathrm{ab}}$ & $133.71^{\mathrm{a}}$ & $107.47^{\text {cdef }}$ \\
\hline & B'0.02+Ca'0.06+Zn'0.03 & $116.99^{\mathrm{abcd}}$ & $94.74^{\mathrm{fg}}$ & $112.53^{\text {bcde }}$ & $87.14^{\mathrm{g}}$ \\
\hline & B'0.08+Ca'1.24 & $128.19^{\mathrm{ab}}$ & $127.05^{\mathrm{ab}}$ & $106.17^{\mathrm{def}}$ & $99.28^{\mathrm{efg}}$ \\
\hline & B’0.17+Ca'1.24 & $115.92^{\text {bcde }}$ & $117.45^{\mathrm{abcd}}$ & $122.55^{\mathrm{abcd}}$ & $118.86^{\mathrm{abcd}}$ \\
\hline \multirow{5}{*}{ Total chlorophyll ${ }^{4}$} & $\mathrm{~B}^{\prime} 0.00+\mathrm{Ca}^{\prime} 0.00$ & $283^{\mathrm{abcd}}$ & $279^{\mathrm{abcd}}$ & $272^{\mathrm{abcd}}$ & $258^{\text {bcde }}$ \\
\hline & $\mathrm{B}^{\prime} 0.02+\mathrm{Ca}^{\prime} 1.24$ & $253^{\text {cdef }}$ & $286^{\mathrm{abc}}$ & $306^{\mathrm{a}}$ & $246^{\text {def }}$ \\
\hline & $\mathrm{B}^{\prime} 0.02+\mathrm{Ca}^{\prime} 0.06+\mathrm{Zn} 0.03$ & $272^{\mathrm{abcd}}$ & $214^{\mathrm{fg}}$ & $258^{\text {bcde }}$ & $201^{\mathrm{g}}$ \\
\hline & B'0.08+Ca'1.24 & $297^{\mathrm{ab}}$ & $285^{\mathrm{abc}}$ & $245^{\text {def }}$ & $228^{\text {efg }}$ \\
\hline & B’0.17+Ca'1.24 & $268^{\mathrm{abcd}}$ & $264^{\text {bcde }}$ & $281^{\mathrm{abcd}}$ & $270^{\mathrm{abcd}}$ \\
\hline \multirow{5}{*}{ Total carotenoids ${ }^{5}$} & $\mathrm{~B}^{\prime} 0.00+\mathrm{Ca}^{\prime} 0.00$ & $63^{\mathrm{abc}}$ & $62^{\mathrm{abcd}}$ & $63^{\mathrm{abcd}}$ & $58^{\text {cdef }}$ \\
\hline & B'0.02+Ca'1.24 & $57^{\text {cdef }}$ & $63^{\mathrm{abc}}$ & $67^{\mathrm{a}}$ & $55^{\text {def }}$ \\
\hline & B'0.02+Ca'0.06+Zn'0.03 & $61^{\mathrm{abcd}}$ & $51^{\mathrm{fg}}$ & $61^{\mathrm{abcd}}$ & $47^{\mathrm{g}}$ \\
\hline & B'0.08+Ca'1.24 & $67^{\mathrm{ab}}$ & $64^{\mathrm{abc}}$ & $55^{\text {def }}$ & $53^{\text {efg }}$ \\
\hline & B'0.17+Ca'1.24 & $60^{\text {bcde }}$ & $56^{\text {cdef }}$ & $62^{\mathrm{abcd}}$ & $62^{\mathrm{abcd}}$ \\
\hline
\end{tabular}

${ }^{1} \mu \mathrm{g} \cdot \mathrm{g}^{-1}$ peel fresh weight, ${ }^{2} p\left(\mathrm{~B}+\mathrm{Ca}{ }^{*} \mathrm{DAFB}\right)=0.0067,{ }^{3} p(\mathrm{~B}+\mathrm{Ca} * \mathrm{DAFB})=0.0050,{ }^{4} p\left(\mathrm{~B}+\mathrm{Ca}^{*} \mathrm{DAFB}\right)=0.005$ and ${ }^{5} p\left(\mathrm{~B}+\mathrm{Ca}^{*} \mathrm{DAFB}\right)=0.0009$.

Table 6. 'Granny Smith' peel photosynthetic pigment ratios and total peroxides.

\begin{tabular}{cccc}
\hline Source & CHLa:CHLb & TCHL:TCAR & TPERO \\
\hline Treatments (B+Ca) & & & \\
B'0.00+Ca'0.00 & $1.29^{\mathrm{ns}}$ & $4.45^{\mathrm{a}}$ & $16.60^{\mathrm{a}}$ \\
B'0.02+Ca'1.24 & 1.29 & $4.49^{\mathrm{a}}$ & $12.44^{\mathrm{b}}$ \\
B'0.02+Ca'0.06+Zn'0.03 & 1.30 & $4.29^{\mathrm{b}}$ & $11.87^{\mathrm{b}}$ \\
B'0.08+Ca'1.24 & 1.29 & $4.42^{\mathrm{a}}$ & $14.66^{\mathrm{ab}}$ \\
B'0.17+Ca'1.24 & 1.28 & $4.50^{\mathrm{a}}$ & $13.21^{\mathrm{b}}$ \\
$p$ & 0.1230 & 0.0081 & 0.0278 \\
Days after full bloom (DAFB) & & & \\
94 & $1.32^{\mathrm{a}}$ & $4.45^{\mathrm{ns}}$ & $10.23^{\mathrm{b}}$ \\
108 & $1.25^{\mathrm{c}}$ & 4.48 & $14.77^{\mathrm{a}}$ \\
122 & $1.30^{\mathrm{b}}$ & 4.42 & $15.77^{\mathrm{a}}$ \\
136 & $1.29^{\mathrm{b}}$ & 4.36 & $14.25^{\mathrm{a}}$ \\
$p$ & 0.0000 & 0.1330 & 0.0011 \\
$p\left(\mathrm{~B}+\mathrm{Ca}^{*} \mathrm{DAFB}\right)$ & 0.4731 & 0.4705 & 0.2979 \\
\hline
\end{tabular}

CHLa:CHLb $=$ Chlorophyll $a$ to Chlorophyll $b$ ratio, TCHL:TCAR $=$ Total Chlorophyll to Total Carotenoids ratio and TPERO $=$ Total Peroxides $\left(\mathrm{nmol} \cdot \mathrm{g}^{-1}\right.$ peel fresh weight $)$. 
The 'Granny Smith' apple peel chlorophyll $a$ to chlorophyll $b$ ratio, total chlorophyll to total carotenoids ratio as well as total peroxides values displayed a significant interaction $\left(\mathrm{B}+\mathrm{Ca}{ }^{*} \mathrm{DAFB}\right)$ effect (Table 6). Foliar $\mathrm{B}+\mathrm{Ca}$ effect was only significant with total chlorophyll to total carotenoids ratio and total peroxides, while fruit maturity (DAFB) effect was only significant with chlorophyll $a$ to chlorophyll $b$ ratio and total peroxides values. With total chlorophyll to total carotenoids ratio, all treatments and the control were not significantly different, except B'0.02+Ca'0.06+Zn'0.03 which was significantly lower compared to all treatments and the control. The highest total peroxides level was recorded with the control, and was significantly different from that of all other treatments, except B'0.08+Ca'1.24. However, total peroxides levels among foliar B+Ca treatments were not significantly different.

As 'Granny Smith' apple fruit maturated, chlorophyll $a$ to chlorophyll $b$ ratio generally decreased, the highest and significantly different level from all other maturity stages was at $94 \mathrm{DAFB}$, and lowest level was at 108 DAFB which differed significantly from the indifferent 122 and 136 DAFB values. However, total peroxides at 94 DAFB was significantly lowest from all other fruit maturity stages, and total peroxides differences at 108, 122 and 136 DAFB periods were not significant.

\subsection{Apple Peel Photosynthetic Efficiency (Fv/Fm Values)}

There were no significant differences among treatments for $\mathrm{Fv} / \mathrm{Fm}$ values in both 'Golden Delicious' and 'Granny Smith' apples at harvest (Table 7). The $\mathrm{Fv} / \mathrm{Fm}$ of sun exposed fruit side (SE) was lower compared to the unexposed fruit side (NSE). Respective standard errors associated with SE and NSE means showed that in 'Golden Delicious' only SE and NSE Fv/Fm was not significantly different in treatment B'0.17+Ca'1.24, yet in 'Granny Smith' all SE and NSE $\mathrm{Fv} / \mathrm{Fm}$ values were significantly different from each other.

Table 7. Apple peel photochemical efficiency (Fv/Fm values).

\begin{tabular}{cccc}
\hline Cultivar & Treatment $(\mathrm{B}+\mathrm{Ca})$ & Fv/Fm SE & Fv/Fm NSE \\
\hline B'0.00+Ca'0.00 & $0.76 \pm 0.03^{*}$ & $0.85 \pm 0.01^{*}$ \\
B'0.02+Ca'1.24 & $0.76 \pm 0.03^{*}$ & $0.83 \pm 0.02^{*}$ \\
'Golden Delicious' & B'0.02+Ca'0.06+Zn'0.03 & $0.80 \pm 0.01^{*}$ & $0.84 \pm 0.01^{*}$ \\
& B'0.08+Ca'1.24 & $0.78 \pm 0.01^{*}$ & $0.86 \pm 0.01^{*}$ \\
B'0.17+Ca'1.24 & $0.81 \pm 0.02$ & $0.86 \pm 0.04$ \\
$p$ & 0.5230 & 0.3230 \\
\hline 'Granny Smith' & B'0.00+Ca'0.00 & $0.81 \pm 0.02^{*}$ & $0.86 \pm 0.01^{*}$ \\
& B'0.02+Ca'1.24 & $0.83 \pm 0.01^{*}$ & $0.86 \pm 0.01^{*}$ \\
& B'0.02+Ca'0.06+Zn'0.03 & $0.83 \pm 0.01^{*}$ & $0.86 \pm 0.01^{*}$ \\
B'0.08+Ca'1.24 & $0.83 \pm 0.01^{*}$ & $0.86 \pm 0.01^{*}$ \\
B'0.17+Ca'1.24 & $0.84 \pm 0.01^{*}$ & $0.87 \pm 0.01^{*}$ \\
$p$ & 0.3390 & 0.9040 \\
\hline
\end{tabular}

$\mathrm{SE}=$ Sun Exposed side of fruit, NSE = Unexposed side of fruit, Fv/Fm = Maximum photochemical efficiency and ${ }^{*}$ significant mean differences between corresponding SE and NSE data. 


\section{Discussion}

\section{1. 'Cripps Pink' Peel Photosynthetic Pigments}

Apple peel biochemistry is strongly influenced by cultivar differences as well as other prevailing factors [45]-[51]. Therefore, in this study each cultivar was considered independently in statistical analyses, with the control (no $\mathrm{B}+\mathrm{Ca}$ ) providing the basic yard-stick to delineate the importance and meaningfulness of the significant differences and effects obtained. In 'Cripps Pink', the significant foliar $\mathrm{B}+\mathrm{Ca}$ effect on all photosynthetic pigment aspects except, chlorophyll $a$ to chlorophyll $b$ ratio and total carotenoids suggests that treatment effect was mainly on chlorophylls and not carotenoids. The significant DAFB effect occurred in patterns agreeable to the physiological apple fruit growth changes, for instance, chlorophyll $a$, chlorophyll $b$ and total chlorophyll levels decreased as fruit maturated, which is expected due to chloroplasts (contain chlorophyll) transitioning to chromoplasts (contain carotenoids) as fruit maturity progresses [52] [53], as well as in apple fruit [54] [55]. Although it appears that 'Cripps Pink' apple peel total carotenoids slightly reduced with advance of DAFB, the total chlorophyll to total carotenoids ratio values with their significant reductions as DAFB advanced, inferred presence of more total carotenoids per unit of total chlorophyll, hence demonstrating that carotenoids were relatively preserved and/or remained constant as total chlorophyll decreased when fruit maturity of 'Cripps Pink' apples progressed.

Evidence of decreasing total chlorophyll on a relatively stable total carotenoids as 'Cripps Pink' fruit maturated is also observed from the significantly increasing chlorophyll $a$ to chlorophyll $b$ ratio values as DAFB progressed. Chloroplasts transitioning to chromoplasts with apple fruit maturation involves chlorophyll destruction, a process preceded by conversion of chlorophyll $b$ to chlorophyll $a$ [56] [57] [58]. The increasing chlorophyll $a$ to chlorophyll $b$ ratio infers that chlorophyll $a$ is higher in comparison to chlorophyll $b$ occurred with advance of DAFB in 'Cripps Pink' apple peel, possibly arising from decompositions of total chlorophyll. The total chlorophyll to total carotenoids ratio estimates the integrity of photosynthetic systems [59], where a higher total chlorophyll to total carotenoids ratio would infer that prerequisites of chlorophyll protection are available, like carotenoids which are more photostable [60] [61]. Carotenoids are heavily involved in antioxidant and photoprotection mechanisms in plants [62] [63] [64], and as well in apple fruit peel tissues [65] [66] [67].

Therefore, the 'Cripps Pink' apple peel total chlorophyll to total carotenoids ratio with $\mathrm{B}^{\prime} 0.02+\mathrm{Ca}^{\prime} 0.06+\mathrm{Zn} 0.03$ in comparison with other treatments, shows reduced photosynthetic integrity due to the significantly low value. However, total chlorophyll to total carotenoids ratio in this $\mathrm{Zn}$-treatment and B'0.02+Ca'1.24 were not significantly different from B'0.00+Ca'0.00 (control), possibly indicating, 1) that $\mathrm{B}^{\prime} 0.08$ and $\mathrm{B}^{\prime} 0.17$ treatments promoted best photosynthetic integrity possibly due to higher $\mathrm{B}$ levels, and 2) a duet function of $\mathrm{B}$ plus $\mathrm{Ca}$ as $\mathrm{B}^{\prime} 0.02+\mathrm{Ca}^{\prime} 1.24$ significantly differed from the $\mathrm{Zn}$ treatment, but not 
from both control, B'0.08 and B'0.17, possibly inferring that more B was necessary in combination with the Ca' 1.24 to promote photosynthetic integrity in the 'Cripps Pink' apple peels. However, propositions 1) and 2) are scantily supported by the peel mineral concentrations, because apple mineral analyses at 80 DAFB (two weeks after the last foliar treatment application), showed that treatment B'0.17+Ca'1.24 had highest B that differed significantly with only the control, but all treatments did not differ [3] [4], yet at fruit harvest treatment $\mathrm{B}^{\prime} 0.02+\mathrm{Ca}^{\prime} 1.24$ peels had highest $\mathrm{B}$ that only significantly differed with the control and B'0.08+Ca'1.24, whereas the Zn, B'0.08 and B'0.17 peels had indifferent B concentration [3] [4].

The incongruity between mineral analyses and peel biochemical effect illustrated above was also observed when these same peel mineral differences did not explain sunburn suppression with these same foliar treatments used in this study [3] [4]. This reveals that there is more of a "biochemical and/or physiological" importance of $\mathrm{B}$, and in combination with $\mathrm{Ca}$ on apple peels that cannot be construed by mere peel tissue differences of one mineral amongst the treatments or control. In the study by [1], B in combination with Ca (duet effect) yielded successful sunburn-browning incidence suppression in 'Golden Delicious' apples, which makes propositions 1) and 2) alluded to above in relation to $\mathrm{B}, \mathrm{Ca}$ variation and their combination in treatments appear valid, and particularly the treatment effects espoused in both 1) and 2) can possibly be related to the sought mode-of-action as photosynthetic systems and chlorophyll destruction precede apple fruit sunburn-browning development.

Overall, treatment $\mathrm{B}^{\prime} 0.02+\mathrm{Ca}^{\prime} 0.06+\mathrm{Zn}^{\prime} 0.03$ showed the lowest chlorophyll $a$, chlorophyll $b$, total chlorophyll and total chlorophyll to total carotenoids ratio levels in 'Cripps Pink' apple peel (Table 2), and these were significantly different from all other foliar B+Ca treatments but not the control. However, the significantly lower total chlorophyll to total carotenoids ratio of this B'0.02+Ca'0.06+Zn'0.03 suggests more preservation of carotenoids against the much decreasing total chlorophyll as fruit maturates, and not an increase in peel carotenoids as treatments did not differ significantly for total carotenoids. However, results associated with B'0.02+Ca'0.06+Zn'0.03 being largely at par with the control (no $\mathrm{B}+\mathrm{Ca}$ ), further indicates that treatment composition has an effect on the 'Cripps Pink' apple peel biochemistry. This is because $\mathrm{Zn}$ inclusion compromised this treatment and not its low Ca levels, the control with no Ca was indifferent with B'0.08+Ca'1.24 for chlorophyll $a$, chlorophyll $b$ and total chlorophyll as well as with $\mathrm{B}^{\prime} 0.02+\mathrm{Ca}^{\prime} 1.24$ for total chlorophyll to total carotenoids ratio.

The observation that B'0.02, B'0.08, B'0.17 all combined with Ca'1.24 not significantly different from each other, demonstrates the possible importance of only B plus $\mathrm{Ca}$ (duet function) free from $\mathrm{Zn}$ to attain stable photosynthetic integrity in contrast to the control for 'Cripps Pink' apple peels. Although there are cases where B'0.02+Ca'1.24 (chlorophyll $b$ and total chlorophyll to total carotenoids ratio) and B'0.08+Ca'1.24 (chlorophyll $a$ and total chlorophyll) were not 
significantly different from the control, it is notable that $\mathrm{B}^{\prime} 0.17+\mathrm{Ca}^{\prime} 1.24$ was consistently significantly different from the control, further confirming the importance of different $\mathrm{B}$ levels in treatment composition, duet importance of appropriate levels of both $\mathrm{B}$ and $\mathrm{Ca}$, and overall discrediting inclusion of $\mathrm{Zn}$. These important observations and differences among these treatments, however, did not cause corresponding differences in sunburn-browning suppression outcomes in 'Cripps Pink' apples [6], suggesting more complex physiological phenomenon with the foliar treatments beyond individual apple peel metabolite dynamics [6].

\section{2. 'Golden Delicious' Peel Photosynthetic Pigments}

Observation on significant foliar B+Ca treatment effect with chlorophyll $a$, total chlorophyll and total carotenoids levels in 'Golden Delicious' apple peels showed that treatment B'0.02+Ca'0.06+Zn'0.03 with significantly lower levels had large importance in the results. Lower $\mathrm{Ca}$ in $\mathrm{B}^{\prime} 0.02+\mathrm{Ca}^{\prime} 0.06+\mathrm{Zn}^{\prime} 0.03$ appears less important in the results as the control (no $\mathrm{B}+\mathrm{Ca}$ ) showed insignificant differences for chlorophyll a, total chlorophyll and total carotenoids with B'0.02, B'0.08 and B'0.17 all with Ca'1.24 treatments. This further suggests that inclusion of $\mathrm{Zn}$ accounts for the B'0.02+Ca'0.06+Zn'0.03 results as was observed in 'Cripps Pink' apple peels as well. Apart from the negative effect of including $\mathrm{Zn}$, no evidence on importance of varying and/or combining B and Ca in all 'Golden Delicious' photosynthetic pigments studied in contrast to 'Cripps Pink' results. This contrast in 'Cripps Pink' and 'Golden Delicious' apples that are genetically related [68], indicates that foliar $\mathrm{B}+\mathrm{Ca}$ treatment formulation and cultivar differences jointly influence respective apple peel biochemical profile, and this may be partly responsible for the varying sunburn-browning suppression outcomes among cultivars and these foliar B+Ca treatments reported by [2] [3] [4].

The significant decrease of the chlorophyll $a$, chlorophyll $b$ and total chlorophyll confirm to decreasing of chlorophyll pigments as apple fruit maturates and as earlier mentioned with 'Cripps Pink'. However, 'Golden Delicious' apple peel, chlorophyll $a$ to chlorophyll $b$ ratio did not show increasing levels as fruit maturated, contrary to 'Cripps Pink', but it is noted that as in 'Cripps Pink', the exact reverse pattern of chlorophyll $a$ to chlorophyll $b$ ratio occurred for total chlorophyll to total carotenoids ratio in 'Golden Delicious' as well as DAFB progressed, further indicating the importance of cultivars differences in the context of this study. The decreasing total carotenoids with advance of DAFB in 'Golden Delicious' is probably due to loss of carotenoids as [69] noted that both chlorophyll and carotenoid pigments decrease in pome fruit where the peel background color changes from green to yellow as fruit maturity progresses. 'Golden Delicious' apples maturate to a carotene-yellow color.

In 'Golden Delicious' apples, the high magnitudes of total flavonoids to total phenolics ratio [7] matched with high magnitudes of total chlorophyll to total carotenoids ratio (Table 3) obtained in this study as the DAFB progressed, 
showing that highest photosynthetic integrity prevailed with highest total flavonoids to total phenolics ratio (more flavonoids per unit of phenolics). This is in line with role of promoting and protecting photostability by flavonoids in plants and as well in fruits [60] [61] [70] [71]. In addition, 'Golden Delicious' apple peel high total chlorophyll to total carotenoids ratio matched with low, chlorophyll $a$ to chlorophyll $b$ ratio and vice versa as DAFB progressed in agreement with photostability analogy illustrated above. Although, high total chlorophyll to total carotenoids ratio matched with low chlorophyll $a$ to chlorophyll $b$ ratio as DAFB advanced in 'Cripps Pink', high total flavonoids to total phenolics ratio values [7], do not correspond to high total chlorophyll to total carotenoids ratio (Table 2). A difference between 'Cripps Pink' and 'Golden Delicious' is thus further observed, and another difference as well with total carotenoids as DAFB advanced, where in 'Cripps Pink' there appears more preservation of total carotenoids as the fruit maturates (Table 2), in contrast to 'Golden Delicious' where the total carotenoids significantly decreases with maturation (Table 3). Carotenoids too have roles in photoprotection mechanisms [62] [63] [64] [72] [73] [74], and may explain the photostability relations in values of total chlorophyll to total carotenoids ratio and chlorophyll $a$ to chlorophyll $b$ ratio in 'Cripps Pink' without a significant total flavonoids to total phenolics ratio input in contrast to 'Golden Delicious' apples. This illustrates that different cultivars have different mechanisms to photostability, and this may as well influence the sought mode-of-action to the extent of involving several domains of peel biochemical aspects [6], overall this is further evidence of cultivar differences in context of this study.

\section{3. 'Granny Smith' Peel Photosynthetic Pigments}

In this study, significant interaction $\left(B+C a^{\star} D A F B\right)$ effect only occurred for 'Granny Smith' apple peel photosynthetic pigments contrary to findings in both 'Cripps Pink' and 'Golden Delicious' apples. However, observation of this significant interaction result reveals it is largely due to, 1) significantly low values associated with $\mathrm{Zn}$ containing treatment across all DAFB levels, which was much clearer in the main effects analysis that is not presented, and 2) B'0.08+Ca' 1.24 that only showed respective pigments decreasing with advancing fruit maturity, but still had insignificant differences with control and most treatments at any DAFB period. Therefore, the importance varying $\mathrm{B}$ and/or $\mathrm{Ca}$ is not realized, but inclusion of $\mathrm{Zn}$ is found not desirable as was the case in 'Cripps Pink' and 'Golden Delicious' apples.

Generally, 'Granny Smith' showed higher magnitudes of chlorophyll and carotenoids compared to both 'Cripps Pink' and 'Golden Delicious', reason being 'Granny Smith' apples maturate to deep green colored fruits unlike both 'Cripps Pink' and 'Golden Delicious'. According to [69], stay-green pome fruit keep higher levels of chlorophyll and carotenoids at advanced maturity, and [61], high levels of chlorophyll and carotenoids prevail together in 'Granny Smith' apples. 
This further underpins the importance of cultivar differences as mentioned earlier. The total chlorophyll to total carotenoids ratio value associated with B'0.02+Ca'0.06+Zn'0.03 was significantly lowered compared to the control (no $\mathrm{B}+\mathrm{Ca}$ ) and all other foliar $\mathrm{B}+\mathrm{Ca}$ treatments, suggesting that inclusion of $\mathrm{Zn}$ compromises this photosynthetic integrity indicator in 'Granny Smith' apple peels. A similar observation was made in both 'Cripps Pink' and 'Golden Delicious' apples. The prevalence of high chlorophylls and carotenoids in 'Granny Smith' peels possibly explains the insignificant DAFB effect with total chlorophyll to total carotenoids ratio levels contrary to findings in both 'Cripps Pink' and 'Golden Delicious' apples.

\subsection{Ineffectiveness of Lipid Peroxidation Assays in Context of This Study}

Photochemical efficiency as measured with Fv/Fm values had insignificant differences among the treatments. According to [75], normal values range from 0.7 to 0.8 which qualifies the values observed in Table 7 as normal, and these insignificant $\mathrm{Fv} / \mathrm{Fm}$ differences among treatments is in agreement with findings of [3] with these treatments. Since, apple fruit susceptibility to oxidative stresses increases with maturity [76] [77] [78], it was envisaged at the inception of this study that peel oxidative stress levels would increase with advance of DAFB, however, that was not observed as total peroxides levels decreased with advance of DAFB in 'Cripps Pink' (Table 2), generally did not decreased or increase within any given treatment with advance of DAFB in both 'Golden Delicious' (Table 4) and 'Granny Smith' (Table 6). This means that the fruit was having literally no oxidative stress to sufficiently impact the photosynthetic systems of the apple peels. Furthermore, these foliar treatments variably associated with significant differences in sunburn-browning in both 'Cripps Pink' and 'Golden Delicious' apples [2] [3] [4], conforming that differential levels of oxidative stresses in apple peels occurred. However, the failure to observe the envisaged behavior of total peroxides levels as alluded to above, and particularly within treatments for 'Cripps Pink' and 'Golden Delicious', raised concerns on suitability of the total peroxides assay for apple peel oxidative stress as used in this study.

Total peroxides content has been reported as a measure of lipid peroxidation and used to indicate oxidative stress status of plant tissues, including apple peels [43] [79]. Total peroxides as quantified following [42] [43] constitutes of $90 \%$ 95\% Hydrogen Peroxide $\left(\mathrm{H}_{2} \mathrm{O}_{2}\right)$, with the remaining 5\% - 10\% being hydroperoxides or peroxy radicals [79] [80]. It was then observed that magnitude of total peroxides levels was generally low in 'Cripps Pink' (red-blushing with maturity), followed by 'Golden Delicious' (carotene-yellowing with maturity) and highest in 'Granny Smith' (green at maturity), meaning that green fruiting had more levels of the quantified $\mathrm{H}_{2} \mathrm{O}_{2}$, which also is the trend of total chlorophyll levels (Table 2, Table 3, and Table 5 all point to a strong possibility that oxidative 
stress measure evaluated in this study largely constituted of $\mathrm{H}_{2} \mathrm{O}_{2}$ generated via the chloroplast pathway. As such, the discussion on the foliar $\mathrm{B}+\mathrm{Ca}$ treatment effect on total peroxides levels as apple peel oxidative stress dynamics was rendered not important in this study, due to the unreliability of the method used.

A key realization from previous study by [81] where no significant differences were found in cell membrane integrity between apple fruit with and without sunburn-browning disorders, raises concern that lipid peroxidation process and its products like total peroxides is very minimal or not there at all in apple peels of fruit with sunburn-browning (lower severity of sunburn) and this was the type of fruit that was utilized in this study. Notably, also the protocol applied in this study has mostly been reported in fruit held in appropriate storage after harvest at a specified maturity stage [42] [43] [79]. However, in this study apple peel evaluations of total peroxides were conducted on consecutively multiple maturing stages, and furthermore, the established variations in peel biochemistry (especially phenolics and ascorbic acid) as apple fruit maturate [82] [83] [84] [85], could introduce quenching effects on respective spectrophotometer absorbance readings [42] [43] reported. However, modifications, particularly extended sample extract dilutions adopted in this study following [86] avoided a quenching effect.

In the studies of [43] [87], oxidative stress in 'Golden Delicious' apple peel was quantified basing on; 1) the thiobarbituric acid assay reactive substances (TBARS) and total peroxides levels both estimated in terms of malondialdehyde (MDA) and $\mathrm{H}_{2} \mathrm{O}_{2}$ levels respectively. Both of these parameters have been used to study oxidative stress levels in plant, fruit as well as apple peel tissues [26] [79] [88] [89] [90]. However, in this study the MDA assays performed following both [43] [87] protocols did not yield trustworthy results as per the spectrophotometer readings obtained. This could have been due to the highly fluctuating sugars and phenolic metabolites that occurs in apple peel tissue with progression of fruit maturity and/or even due to fruit exposure to solar irradiance as samples were collected from outer tree canopy positions [45] [84] [91] [92]. Sugars and phenolic metabolites are abundant in apple peels [82] [83] [85], and are well known to compromise MDA assay output [87] [93]. Overall, apple fruit peel tissue oxidative stress analysis using the MDA or total peroxides assay seems best suited for studies dealing with single maturity stage, and mature harvested fruit in storage as was the case in the studies of both [43] [79], but most importantly lipid peroxidation assays cannot determine oxidative stress levels in apple fruit with sunburn-browning in line with findings of [81] as well.

Probably future endeavors in relation to the context of this study, should consider using confocal microscopic analyses of oxidative stress species [94] [95] [96] or non-destructive hyperspectral imaging [97]. Oxidative stress in plant tissues can arise from enzymatic and non-enzymatic pathways, whereby enzymatic source is via catalytic activities of lipoxygenases (EC 1.13.11.12), while non-enzymatic sources are due to reactive oxygen species (ROS) formed 
within different cell compartments [98] [99]. Lipoxygenase-derived oxidative stress is more associated with plant organ senescence [100] [101], although non-enzymatic oxidative stress was reported to be involved as well [102]. However, an important aspect in relation to this study, is that oxidative stress species and/or intermediates generated in the lipoxygenase-enzymatic pathway are deactivated in respective complex enzymatic processes while those generated in non-enzymatic pathway are not subjected to enzymatic deactivation [103], which exacerbates their effects on cellular physiology [28].

Following from above, non-enzymatic oxidative stress is expected to predominate apple fruit sunburn-browning phenomenon as the respective plant organ (the apple fruit) is certainly not in senescence, even up to commercial harvest, which is in agreement with previous studies of [34] [104] [105]. Notably, ascorbic acid and related cycle including glutathione are part of the non-enzymatic antioxidant which are very important in scavenging of ROS [106], however, prior to this study preliminary experiments data with such foliar treatments did not yield significant differences for ascorbate parameters and glutathione as well (E. Lotze and E.W. Hoffman, Stellenbosch University, Unpublished data, 2014). This suggests that apple peel biochemicals like flavonoids which protect cell organelles with high metabolic activities [70] [71], and are as well very potent antioxidants seem more versatile in curtailing oxidative stress inducers of sunburn-browning in line with recommendation of deeper peel flavonoids investigations made by [7], as well as in agreement with studies of [104] [105] [107].

\section{Conclusion}

Post-full-bloom foliar boron plus calcium applications affect photosynthetic pigments in apple peels depending on treatment formulation and cultivar idiosyncrasies. For instance, inclusion of $\mathrm{Zn}$ lowered the levels of apple peel photosynthetic pigment attributes in all the study cultivars, yet varying $\mathrm{B}$, Ca levels in treatments showed that a B plus Ca duet function was only important in 'Cripps Pink'. Although significant treatment and interaction effects were recorded in 'Golden Delicious' and 'Granny Smith' apple peels respectively, treatment composition in relation to different B or Ca levels could not appreciably explain any effect on the photosynthetic pigment attributes in these two cultivars, the inclusion of $\mathrm{Zn}$-treatment caused significant changes in respective results. Remarkably, only photosynthetic integrity indicators of 'Golden Delicious' related with levels of flavonoids found in a preceding study, providing another proof of cultivar differences in relation to this study. The study cannot confirm precisely that foliar $\mathrm{B}+\mathrm{Ca}$ treatments reduce oxidative stress levels in the studied apples towards their harvest maturity. This resulted from the inadequacy or low efficiency of lipid peroxidation assays to yield oxidative stress differences under the experimental circumstances and conditions of this study. However, this finding does not negate the notion that apple fruit oxidative stress increases with maturity, or even role of oxidative stress in development of sun- 
burn-browning as strongly established in literature, but rather this study highlights the need to find alternative approaches to lipid peroxidation assays and preferably non-destructive methods in investigating peel oxidative stress dynamics in maturating apple fruit.

\section{Acknowledgements}

Support for this research was obtained from Nulandis (Pty), South Africa and also through a capacity building competitive grant (RU/2015/DRRG/01/004) provided by Regional Universities Forum for Capacity Building in Agriculture (RUFORUM) to Makerere and Stellenbosch Universities.

\section{Conflicts of Interest}

The authors declare no conflicts of interest regarding the publication of this paper.

\section{References}

[1] Lötze, E. and Hoffman, E.W. (2014) Foliar Application of Calcium plus Boron Reduces the Incidence of Sunburn in 'Golden Delicious' Apple. Journal of Horticultural Science and Biotechnology, 89, 607-612. https://doi.org/10.1080/14620316.2014.11513127

[2] Lötze, E., Daiber, S.H. and Midgley, S.J.E. (2017) Boron in Combination with Calcium Reduces Sunburn in Apple Fruit. Boron, 2, 123-127.

[3] Daiber, S.H. (2017) Quantifying Changes in Tree Physiology after Amelioration to Reduce Sunburn on Apples. MSc Thesis, Stellenbosch University, Stellenbosch.

[4] Lötze, E., Daiber, S.H. and Midgley, S.J.E. (2018) Evaluating the Efficacy of a Preharvest Combination of Calcium and Boron as Foliar Application to Reduce Sunburn on 'Cripps Pink' Apples. Acta Horticulture, 1217, 61-68. https://doi.org/10.17660/ActaHortic.2018.1217.7

[5] Mwije, A. (2017) Pioneering a New Paradigm of Sunburn Mitigation in Apples. In: Withers, J., Ed., Bringing Science to Communities: Voices from the Field (Issue 1), A RUFORUM Publication, Kampala, 20-23.

[6] Mwije, A. (2020) A Study of the Dynamics of Sunburn Reduction in Apple (Malus domestica) Using Foliar Applications of a Combination of Boron and Calcium. Ph.D. Thesis, Stellenbosch University, South Africa.

[7] Mwije, A., Hoffman, E.W. and Lötze, E. (2020) Apple Peel Biochemical Changes after Foliar Application of Boron Combined with Calcium I. Phenolics and Physico-Chemical Attributes. American Journal of Plant Sciences, 11, 965-986.

[8] Felicetti, D.A. and Schrader, L.E. (2009) Changes in Pigment Concentrations Associated With Sunburn Browning of Five Apple Cultivars. II. Phenolics. Plant Science, 176, 84-89. https://doi.org/10.1016/j.plantsci.2008.09.010

[9] Felicetti, D.A. and Schrader, L.E. (2009) Changes in Pigment Concentrations Associated With Sunburn Browning of Five Apple Cultivars. I. Chlorophylls and Carotenoids. Plant Science, 176, 78-83. https://doi.org/10.1016/j.plantsci.2008.09.013

[10] Wünsche, J.N., Greer, D.H., Palmer, J.W., Lang, A. and McGhie, T. (2001) Sunburn-The Cost of a High Light Environment. Acta Horticulturae, 557, 349-356. https://doi.org/10.17660/ActaHortic.2001.557.46 
[11] Wünsche, J.N., Lombardini, L. and Greer, D.H. (2004) "Surround" Particle Film Applications-Effects on Whole Canopy Physiology of Apple. Acta Horticulturae 636, 565-571. https://doi.org/10.17660/ActaHortic.2004.636.72

[12] Schrader, L., Sun, J., Zhang, J., Felicetti, D. and Tian, J. (2008) Heat and Light-Induced Apple Skin Disorders: Causes and Prevention. Acta Horticulturae, 772, 51-58. https://doi.org/10.17660/ActaHortic.2008.772.5

[13] Vrettos, J.S., Limburg, J. and Brudvig, G.W. (2001) Mechanism of Photosynthetic Water Oxidation: Combining Biophysical Studies of Photosystem II with Inorganic Model Chemistry. Biochimica et Biophysica Acta, 1503, 229-245. https://doi.org/10.1016/S0005-2728(00)00214-0

[14] Yachandra, V.K. and Yano, J. (2011) Calcium in the Oxygen-Evolving Complex: Structural and Mechanistic Role Determined by X-Ray Spectroscopy. Journal of Photochemistry and Photobiology B: Biology, 104, 51-59. https://doi.org/10.1016/j.jphotobiol.2011.02.019

[15] Yang, S., Wang, F., Guo, F., Meng, J.J., Li, X.G. and Wan, S.B. (2015) Calcium Contributes to Photoprotection and Repair of Photosystem II in Peanut Leaves during Heat and High Irradiance. Journal of Integrative Plant Biology, 57, 486-495. https://doi.org/10.1111/jipb.12249

[16] Jiang, Y. and Huang, B. (2001) Effects of Calcium on Antioxidant Activities and Water Relations Associated with Heat Tolerance in Two Cool-Season Grasses. Journal of Experimental Botany, 52, 341-349. https://doi.org/10.1093/jexbot/52.355.341

[17] Seth, K. and Aery, N.C. (2014) Effect of Boron on the Contents of Chlorophyll, Carotenoid, Phenol and Soluble Leaf Protein in Mung Bean, Vigna radiata (L.) Wilczek. Proceedings of National Academy of Sciences, India Section B: Biological Sciences, 84, 713-719. https://doi.org/10.1007/s40011-013-0293-4

[18] Ganie, M.A., Akhter, F., Bhat, M.A., Malik, A.R., Junaid, J.M., Shah, M.A., Bhat, A.H. and Bhat, T.A. (2013) Boron-A Critical Nutrient Element for Plant Growth and Productivity with Reference to Temperate Fruits. Current Science, 104, 76-85.

[19] Kobayashi, M., Nakagawa, H., Asaka, T. and Matoh, T. (1999) Borate Rhamnogalacturonan II Bonding Reinforced by $\mathrm{Ca}^{2+}$ Retains Pectic Polysaccharides in Higher-Plant Cell Walls. Plant Physiology, 119, 199-204. https://doi.org/10.1104/pp.119.1.199

[20] Bolanos, L., Lukaszewski, K., Bonilla, I. and Blevins, D. (2004) Why Boron? Plant Physiology and Biochemistry, 42, 907-912. https://doi.org/10.1016/j.plaphy.2004.11.002

[21] Camacho-Cristobal, J.J., Herrera-Rodriguez, M.B., Beato, V.M., Rexach, J., Navarro-Gochicoa, M.T., Maldonado, J.M. and Gonzalez-Fontes, A. (2008) The Expression of Several Cell Wall-Related Genes in Arabidopsis Roots is Down-Regulated under Boron Deficiency. Environmental and Experimental Botany, 63, 351-358. https://doi.org/10.1016/j.envexpbot.2007.12.004

[22] Verma, S. and Dubey, R.S. (2003) Lead Toxicity Induces Lipid Peroxidation and Alters the Activities of Antioxidant Enzymes in Growing Rice Plants. Plant Science, 164, 645-655. https://doi.org/10.1016/S0168-9452(03)00022-0

[23] Skorzynska-Polit, E. 2007. Lipid Peroxidation in Plant Cells, Its Physiological Role and Changes under Heavy Metal Stress. Acta Societatis Botanicorum Poloniae, 76, 49-54. https://doi.org/10.5586/asbp.2007.006

[24] Xu, S., Li, J., Zhang, X., Wei, H. and Cui, L. (2006) Effects of Heat Acclimation Pretreatment on Changes of Membrane Lipid Peroxidation, Antioxidant Metabolites, 
and Ultrastructure of Chloroplasts in Two Cool-Season Turfgrass Species under Heat Stress. Environmental and Experimental Botany, 56, 274-285. https://doi.org/10.1016/j.envexpbot.2005.03.002

[25] Jiang, Y. and Huang, B. (2001) Drought and Heat Stress Injury to Two Cool-Season Turfgrasses in Relation to Antioxidant Metabolism and Lipid Peroxidation. Crop Science, 41, 436-442. https://doi.org/10.2135/cropsci2001.412436x

[26] Song, X., Wang, Y. and Lv, X. (2016) Responses of Plant Biomass, Photosynthesis and Lipid Peroxidation to Warming and Precipitation Change in Two Dominant Species (Stipa grandis and Leymus chinensis) from North China Grasslands. Ecology and Evolution, 6, 1871-1882. https://doi.org/10.1002/ece3.1982

[27] Agarwal, S., Sairam, R.K., Srivastava, G.C., Tyagi, A. and Meena, R.C. (2005) Role of ABA, Salicylic Acid, Calcium and Hydrogen Peroxide on Antioxidant Enzymes Induction in Wheat Seedlings. Plant Science, 169, 559-570.

https://doi.org/10.1016/j.plantsci.2005.05.004

[28] Gill, S.S. and Tuteja, N. (2010) Reactive Oxygen Species and Antioxidant Machinery in Abiotic Stress Tolerance in Crop Plants. Plant Physiology and Biochemistry, 48, 909-930. https://doi.org/10.1016/j.plaphy.2010.08.016

[29] Karuppanapandian, T., Moon, J.C., Kim, C., Manoharan, K. and Kim, W. (2011) Reactive Oxygen Species in Plants: Their Generation, Signal Transduction, and Scavenging Mechanisms. Australian Journal of Crop Science, 5, 709-725.

[30] Chen, L.S., Li, P. and Cheng, L. (2008) Effects of High Temperature Coupled with High Light on the Balance between Photooxidation and Photoprotection in the Sun-Exposed Peel of Apple. Planta, 228, Article No.: 745.

https://doi.org/10.1007/s00425-008-0776-3

[31] Chen, L.-S., Li, P. and Cheng, L. (2009) Comparison of Thermotolerance of Sun-Exposed Peel and Shaded Peel of "Fuji" Apple. Environmental and Experimental Botany, 66, 110-116. https://doi.org/10.1016/j.envexpbot.2008.12.017

[32] Yuri, J.A., Neira, A., Quilodran, A., Razmilic, I., Motomura, Y., Torres, C. and Palomo, I. (2010) Sunburn on Apples is Associated with Increases in Phenolic Compounds and Antioxidant Activity as a Function of the Cultivar and Areas of the Fruit. Journal of Food, Agriculture and Environment, 8, 920-925.

[33] Yuri, J.A., Neira, A., Maldonado, F., Quilodran, A., Simeone, D., Razmilic, I. and Palomo, I. (2014) Total Phenol and Quercetin Content and Antioxidant Activity in Apples in Response to Thermal, Light Stress and to Organic Management. Journal of Applied Botany and Food Quality, 87, 131-138.

[34] Zhang, J., Niu, J., Duan, Y., Zhang, M., Liu, J., Li, P. and Ma, F. (2015) Photoprotection Mechanism in the "Fuji" Apple Peel at Different Levels of Photooxidative Sunburn. Physiologia Plantarum, 154, 54-65. https://doi.org/10.1111/ppl.12272

[35] Policarpo, M., Di Marco, L., Farina, V. and Tagliavini, S. (2002) Effect of Foliar Nutrition on Peach (Prunus persica L. Batsch) Yield and Fruit Quality as Related to Different Crop Loads. Acta Horticulturae, 594, 659-666.

https://doi.org/10.17660/ActaHortic.2002.594.89

[36] Garc, M.F., Hern, J.A., Lopez-Gomez, E., San Juan, M.A., Diaz-Vivancos, P., Mataix Beneyto, J., Garcia-Legaz, M.F. and Hernandez, J.A. (2007) Effect of Rootstocks Grafting and Boron on the Antioxidant Systems and Salinity Tolerance of Loquat Plants (Eriobotrya japonica Lindl.). Environmental and Experimental Botany, 60, 151-158. https://doi.org/10.1016/j.envexpbot.2006.10.007

[37] Thurzo, S., Szabo, Z., Nyeki, J., Nagy, P.T., Silva, A.P. and Goncalves, B. (2010) Effect of Boron and Calcium Sprays on Photosynthetic Pigments, Total Phenols and 
Flavonoid Content of Sweet Cherry (Prunus avium L.). Acta Horticulturae, 868, 457-462. https://doi.org/10.17660/ActaHortic.2010.868.64

[38] Singh, D.P., Beloy, J., Mcinerney, J.K. and Day, L. (2012) Impact of Boron, Calcium and Genetic Factors on Vitamin C, Carotenoids, Phenolic Acids, Anthocyanins and Antioxidant Capacity of Carrots (Daucus carota). Food Chemistry, 132, 1161-1170. https://doi.org/10.1016/j.foodchem.2011.11.045

[39] Sakhonwasee, S. and Phingkasan, W. (2017) Effects of the Foliar Application of Calcium on Photosynthesis, Reactive Oxygen Species Production, and Changes in Water Relations in Tomato Seedlings under Heat Stress. Horticulture Environment and Biotechnology, 58, 119-126. https://doi.org/10.1007/s13580-017-0194-1

[40] Lichtenthaler, H.K. (1987) Chlorophylls and Carotenoids: Pigments of Photosynthetic Biomembranes. Methods in Enzymology, 148, 350-382.

https://doi.org/10.1016/0076-6879(87)48036-1

[41] Lichtenthaler, H.K. and Buschmann, C. (2001) Chlorophylls and Carotenoids: Measurement and Characterization by UV-VIS Spectroscopy. Current Protocols in Food Analytical Chemistry, 1, F4.3.1-F4.3.8. https://doi.org/10.1002/0471142913.faf0403s01

[42] Brennan, T. and Frenkel, C. (1977) Involvement of Hydrogen Peroxide in the Regulation of Senescence in Pear. Plant Physiology, 59, 411-416.

https://doi.org/10.1104/pp.59.3.411

[43] Du, Z. and Bramlage, W.J. (1995) Peroxidative Activity of Apple Peel in Relation to Development of Poststorage Disorders. HortScience, 30, 205-209. https://doi.org/10.21273/HORTSCI.30.4.205

[44] Statistica Software (2019). https://www.tibco.com/products/data-science

[45] Piskolczi, M., Varga, C. and Racskó, J. (2004) A Review of the Meteorological Causes of Sunburn Injury on the Surface of Apple Fruit (Malus domestica Borkh). Journal of Fruit and Ornamental Plant Research, 12, 245-252.

[46] Mainla, L., Moor, U., Karp, K. and Pussa, T. (2011) The Effect of Genotype and Rootstock on Polyphenol Composition of Selected Apple Cultivars in Estonia. Zemdirbyste-Agriculture, 98, 63-70.

[47] Kviklys, D., Liaudanskas, M., Janulis, V., Viskelis, P., Rubinskiene, M., Lanauskas, J. and Uselis, N. (2014) Rootstock Genotype Determines Phenol Content in Apple Fruits. Plant, Soil and Environment, 60, 234-240. https://doi.org/10.17221/71/2014-PSE

[48] Feng, F., Li, M., Ma, F. and Cheng, L. (2014) The Effects of Bagging and Debagging on External Fruit Quality, Metabolites, and the Expression of Anthocyanin Biosynthetic Genes in "Jonagold” Apple (Malus domestica Borkh.). Scientia Horticulturae, 165, 123-131. https://doi.org/10.1016/j.scienta.2013.11.008

[49] Feng, F., Li, M., Ma, F. and Cheng, L. (2014) Effects of Location Within the Tree Canopy on Carbohydrates, Organic Acids, Amino Acids and Phenolic Compounds in the Fruit Peel and Flesh from Three Apple (Malus $\times$ domestica) Cultivars. Hor ticulture Research, 1, Article No.: 14019. https://doi.org/10.1038/hortres.2014.19

[50] De Paepe, D., Valkenborg, D., Noten, B., Servaes, K., Diels, L., De Loose, M., Van Droogenbroeck, B. and Voorspoels, S. (2015) Variability of the Phenolic Profiles in the Fruits From Old, Recent and New Apple Cultivars Cultivated in Belgium. Metabolomics, 11, 739-752.

[51] Le Bourvellec, C., Bureau, S., Renard, C.M.G.C., Plenet, D., Gautier, H., Touloumet, L., Girard, T. and Simon, S. (2015) Cultivar and Year Rather than Agricultural Practices Affect Primary and Secondary Metabolites in Apple Fruit. PLoS ONE, 10, 
e0141916. https://doi.org/10.1371/journal.pone.0141916

[52] Bian, W., Barsan, C., Egea, I., Purgatto, E., Chervin, C., Zouine, M., Latche, A., Bouzayen, M. and Pech, J.C. (2011) Metabolic and Molecular Events Occurring During Chromoplast Biogenesis. Journal of Botany, 2011, Article ID: 289859. https://doi.org/10.1155/2011/289859

[53] Egea, I., Bian, W., Barsan, C., Jauneau, A., Pech, J.C., Latche, A., Li, Z. and Chervin, C. (2011) Chloroplast to Chromoplast Transition in Tomato Fruit: Spectral Confocal Microscopy Analyses of Carotenoids and Chlorophylls in Isolated Plastids and Time-Lapse Recording on Intact Live Tissue. Annals of Botany, 108, 291-297. https://doi.org/10.1093/aob/mcr140

[54] Delgado-Pelayo, R., Gallardo-Guerrero, L. and Hornero-Mendez, D. (2014) Chlorophyll and Carotenoid Pigments in the Peel and Flesh of Commercial Apple Fruit Varieties. Food Research International, 65, 272-281. https://doi.org/10.1016/j.foodres.2014.03.025

[55] Merzlyak, M.N. (2006) Modeling Pigment Contributions to Spectral Reflection of Apple Fruit. Photochemical \& Photobiological Sciences, 5, 748-754. https://doi.org/10.1039/b602160c

[56] Kim, E.H., Li, X.P., Razeghifard, R., Anderson, J.M., Niyogi, K.K., Pogson, B.J. and Chow, W.S. (2009) The Multiple Roles of Light-Harvesting Chlorophyll a/b-Protein Complexes Define Structure and Optimize Function of Arabidopsis Chloroplasts: A Study Using Two Chlorophyll b-Less Mutants. Biochimica et Biophysica Acta, 1787, 973-984. https://doi.org/10.1016/j.bbabio.2009.04.009

[57] Hortensteiner, S. and Krautler, B. (2011) Chlorophyll Breakdown in Higher Plants. Biochimica et Biophysica Acta, 1807, 977-988.

https://doi.org/10.1016/j.bbabio.2010.12.007

[58] Tanaka, R. and Tanaka, A. (2011) Chlorophyll Cycle Regulates the Construction and Destruction of the Light-Harvesting Complexes. Biochimica et Biophysica Acta, 1807, 968-976. https://doi.org/10.1016/j.bbabio.2011.01.002

[59] Hannoufa, A. and Hossain, Z. (2012) Regulation of Carotenoid Accumulation in Plants. Biocatalysis and Agricultural Biotechnology, 1, 198-202. https://doi.org/10.1016/j.bcab.2012.03.004

[60] Havaux, M. (1998) Carotenoids as Membrane Stabilizers in Chloroplasts. Trends in Plant Science, 3, 147-151. https://doi.org/10.1016/S1360-1385(98)01200-X

[61] Ampomah-Dwamena, C., Dejnoprat, S., Lewis, D., Sutherland, P., Volz, R.K. and Allan, A.C. (2012) Metabolic and Gene Expression Apple (Malus $\times$ domestica) Carotenogenesis. Journal of Experimental Botany, 63, 4497-4511. https://doi.org/10.1093/jxb/ers134

[62] Racchi, M.L. (2013) Antioxidant Defenses in Plants with Attention to Prunus and Citrus spp. Antioxidants, 2, 340-369. https://doi.org/10.3390/antiox2040340

[63] Bandurska, H., Niedziela, J. and Chadzinikolau, T. (2013) Separate and Combined Responses to Water Deficit and UV-B Radiation. Plant Science, 213, 98-105. https://doi.org/10.1016/j.plantsci.2013.09.003

[64] Nisar, N., Li, L., Lu, S., Khin, N.C. and Pogson, B.J. (2015) Carotenoid Metabolism in Plants. Molecular Plant, 8, 68-82. https://doi.org/10.1016/j.molp.2014.12.007

[65] Merzlyak, M.N. and Solovchenko, A.E. (2002) Photostability of Pigments in Ripening Apple Fruit: A Possible Photoprotective Role of Carotenoids during Plant Senescence. Plant Science, 163, 881-888.

https://doi.org/10.1016/S0168-9452(02)00241-8 
[66] Merzlyak, M.N., Solovchenko, A.E. and Chivkunova, O.B. (2002) Patterns of Pigment Changes in Apple Fruits during Adaptation to High Sunlight and Sunscald Development. Plant Physiology and Biochemistry, 40, 679-684. https://doi.org/10.1016/S0981-9428(02)01408-0

[67] Felicetti, D.A. and Schrader, L.E. (2008) Changes in Pigment Concentrations Associated with the Degree of Sunburn Browning of "Fuji" Apple. Journal of the American Society of Horticultural Science, 133, 27-34. https://doi.org/10.21273/JASHS.133.1.27

[68] Cripps, J.E.L., Richards, L.A. and Mairata, A.M. (1993) "Pink Lady" Apple. HortScience, 28, 1057. https://doi.org/10.21273/HORTSCI.28.10.1057

[69] Charoenchongsuk, N., Ikeda, K., Itai, A., Oikawa, A. and Murayama, H. (2015) Comparison of the Expression of Chlorophyll-Degradation-Related Genes During Ripening Between Stay-Green and Yellow-Pear Cultivars. Scientia Horticulturae, 181, 89-94. https://doi.org/10.1016/j.scienta.2014.10.005

[70] Agati, G., Azzarello, E., Pollastri, S. and Tattini, M. (2012) Flavonoids as Antioxidants in Plants: Location and Functional Significance. Plant Science, 196, 67-76. https://doi.org/10.1016/j.plantsci.2012.07.014

[71] Agati, G., Brunetti, C., Ferdinando, M.D., Ferrini, F., Pollastri, S. and Tattini, M. (2013) Functional Roles of Flavonoids in Photoprotection: New Evidence, Lessons from the Past. Plant Physiology and Biochemistry, 72, 35-45. https://doi.org/10.1016/j.plaphy.2013.03.014

[72] Dellapenna, D. (1999) Carotenoid Synthesis and Function in Plants: Insights from Mutant Studies in Arabidopsis. Pure and Applied Chemistry, 71, 2205-2212. https://doi.org/10.1351/pac199971122205

[73] Havaux, M. and Niyogi, K.K. (1999) The Violaxanthin Cycle Protects Plants from Photooxidative Damage by More Than One Mechanism. Proceedings of the National Academy of Sciences of the United States of America, 96, 8762-8767. https://doi.org/10.1073/pnas.96.15.8762

[74] Mu, P., Li, X.P. and Niyogi, K.K. (2001) Update on Photosynthesis Non Photochemical Quenching. A Response to Excess Light Energy. Plant Physiology, 125, 1558-1566. https://doi.org/10.1104/pp.125.4.1558

[75] Ritchie, G.A. (2006) Chlorophyll Fluorescence: What Is It and What Do the Numbers Mean? National Proceedings. Forest and Conservation Nursery Associations, RMRS-P-43, 34-43.

[76] Dussi, M.C., Giardina, G. and Reeb, P. (2005) Shade Nets Effect on Canopy Light Distribution and Quality of Fruit and Spur Leaf on Apple cv. Fuji. Spanish Journal of Agricultural Research, 3, 253-260. https://doi.org/10.5424/sjar/2005032-144

[77] Fan, L., Song, J., Forney, C.F. and Jordan, M.A. (2011) Fruit Maturity Affects the Response of Apples to Heat Stress. Postharvest Biology and Technology, 62, 35-42. https://doi.org/10.1016/j.postharvbio.2011.04.007

[78] Racskó, J. and Schrader, L.E. (2012) Sunburn of Apple Fruit: Historical Background, Recent Advances and Future Perspectives. Critical Reviews in Plant Sciences, 31, 455-504. https://doi.org/10.1080/07352689.2012.696453

[79] Rao, M. V, Watkins, C.B., Brown, S.K. and Weeden, N.F. (1998) Active Oxygen Species Metabolism in "White Angel” $\times$ "Rome Beauty" Apple Selections Resistant and Susceptible to Superficial Scald. Journal of the American Society for Horticultural Science, 123, 299-304. https://doi.org/10.21273/JASHS.123.2.299

[80] Blokhina, O., Virolainen, E. and Fagerstedt, K.V. (2003) Antioxidants, Oxidative Damage and Oxygen Deprivation Stress: A Review. Annals of Botany, 91, 179-194. 
https://doi.org/10.1093/aob/mcf118

[81] Schrader, L.E., Zhang, J. and Duplaga, W.K. (2001) Two Types of Sunburn in Apple Caused by High Fruit Surface (Peel) Temperature. Plant Health Progress, 2, No. 1. https://doi.org/10.1094/PHP-2001-1004-01-RS

[82] Wolfe, K., Wu, X. and Liu, R.H. (2003) Antioxidant Activity of Apple Peels. Journal of Agricultural and Food Chemistry, 51, 609-614. https://doi.org/10.1021/jf020782a

[83] Tsao, R., Yang, R., Xie, S., Sockovie, E. and Khanizadeh, S. (2005) Which Polyphenolic Compounds Contribute to the Total Antioxidant Activities of Apple? Journal of Agricultural and Food Chemistry, 53, 4989-4995. https://doi.org/10.1021/jf048289h

[84] Moyle, C.W.A. (2011) Polyphenols in Apples and Their Interactions with Vascular Endothelial Cells. PhD Dissertation, University of East Anglia, Norwich.

[85] Kalinowska, M., Bielawska, A., Lewandowska-Siwkiewicz, H., Priebe, W. and Lewandowski, W. (2014) Apples: Content of Phenolic Compounds vs. Variety, Part of Apple and Cultivation Model, Extraction of Phenolic Compounds, Biological Properties. Plant Physiology and Biochemistry, 84, 169-188. https://doi.org/10.1016/j.plaphy.2014.09.006

[86] Perez, F.J. and Rubio, S. (2006) An Improved Chemiluminescence Method for Hydrogen Peroxide Determination in Plant Tissues. Plant Growth Regulation, 48, 89-95. https://doi.org/10.1007/s10725-005-5089-y

[87] Du, Z. and Bramlage, W.J. (1992) Modified Thiobarbituric Acid Assay for Measuring Lipid Oxidation in Sugar-Rich Plant Tissue Extracts. Journal of Agricultural and Food Chemistry, 40, 1566-1570. https://doi.org/10.1021/jf00021a018

[88] Mishra, R.K. and Singhal, G.S. (1992) Function of Photosynthetic Apparatus of Intact Wheat Leaves under High Light and Heat Stress and its Relationship with Peroxidation of Thylakoid Lipids. Plant Physiology, 98, 1-6.

https://doi.org/10.1104/pp.98.1.1

[89] Vilaplana, R., Valentines, M.C., Toivonen, P. and Larrigaudiere, C. (2006) Antioxidant Potential and Peroxidative State of "Golden Smoothee" Apples Treated with 1-Methylcyclopropene. Journal of the American Society for Horticultural Science, 131, 104-109. https://doi.org/10.21273/JASHS.131.1.104

[90] Duan, X., Liu, T., Zhang, D., Su, X., Lin, H. and Jiang, Y. (2011) Effect of Pure Oxygen Atmosphere on Antioxidant Enzyme and Antioxidant Activity of Harvested Litchi Fruit during Storage. Food Research International, 44, 1905-1911. https://doi.org/10.1016/j.foodres.2010.10.027

[91] Bakhshi, D. and Arakawa, O. (2006) Induction of Phenolic Compounds Biosynthesis with Light Irradiation in the Flesh of Red and Yellow Apples. Journal of Applied Horticulture, 8, 101-104. https://doi.org/10.37855/jah.2006.v08i02.23

[92] Jakopic, J., Stampar, F. and Veberic, R. (2009) The Influence of Exposure to Light on the Phenolic Content of 'Fuji' Apple. Scientia Horticulturae, 123, 234-239. https://doi.org/10.1016/j.scienta.2009.09.004

[93] Hodges, D.M., Delong, J.M., Forney, C.F. and Prange, R.K. (2015) Improving the Thiobarbituric Acid-Reactive-Substances Assay for Estimating Lipid Peroxidation in Plant Tissues Containing Anthocyanin and Other Interfering Compounds. Planta, 207, 604-611. https://doi.org/10.1007/s004250050524

[94] Macarisin, D., Cohen, L., Eick, A., Rafael, G., Belausov, E., Wisniewski, M. and Droby, S. (2007) Penicillium digitatum Suppresses Production of Hydrogen Peroxide in Host Tissue during Infection of Citrus Fruit. Postharvest Pathology and Mycotoxins, 97, 1491-1500. https://doi.org/10.1094/PHYTO-97-11-1491 
[95] Sabban-Amin, R., Feygenberg, O., Belausov, E. and Pesis, E. (2011) Low Oxygen and 1-MCP Pretreatments Delay Superficial Scald Development by Reducing Reactive Oxygen Species (ROS) Accumulation in Stored 'Granny Smith' Apples. Postharvest Biology and Technology, 62, 295-304. https://doi.org/10.1016/j.postharvbio.2011.06.016

[96] Mditshwa, A., Fawole, O.A., Vries, F., van Der Merwe, K., Crouch, E. and Opara, U.L. (2016) Classification of 'Granny Smith' Apples with Different Levels of Superficial Scald Severity Based on Targeted Metabolites and Discriminant Analysis. Journal of Applied Botany and Food Quality, 89, 49-55.

[97] Kong, W., Liu, F., Zhang, C., Zhang, J. and Feng, H. (2016) Non-Destructive Determination of Malondialdehyde (MDA) Distribution in Oilseed Rape Leaves by Laboratory Scale NIR Hyperspectral Imaging. Scientific Reports, 6, Article No.: 35393. https://doi.org/10.1038/srep35393

[98] Axelrod, B., Cheesbrough, T.M. and Laakso, S. (1981) Lipoxygenase from Soybeans: EC 1.13.11.12 Linoleate: Oxygen Oxidoreductase. Methods in Enzymology, 71, 441-451. https://doi.org/10.1016/0076-6879(81)71055-3

[99] Dix, T.A. and Aikens, J. (1993) Mechanisms and Biological Relevance of Lipid Peroxidation Initiation. Chemical Research in Toxicology, 6, 2-18. https://doi.org/10.1021/tx00031a001

[100] Lynch, D.V. and Thompson, J.E. (1984) Lipoxygenase-Mediated Production of Superoxide Anion in Senescing Plant Tissue. FEBS Letters, 173, 251-254. https://doi.org/10.1016/0014-5793(84)81057-1

[101] He, Y., Fukushige, H., Hildebrand, D.F. and Gan, S. (2002) Evidence Supporting a Role of Jasmonic Acid in Arabidopsis Leaf Senescence. Plant Physiology, 128, 435-441. https://doi.org/10.1104/pp.010843

[102] Berger, S., Weichert, H., Porzel, A., Wasternack, C., Kuhn, H. and Feussner, I. (2001) Enzymatic and Non-Enzymatic Lipid Peroxidation in Leaf Development. Biochimica et Biophysica Acta, 1533, 266-276. https://doi.org/10.1016/S1388-1981(01)00161-5

[103] Spiteller, G. (2003) The Relationship Between Changes in the Cell Wall, Lipid Peroxidation, Proliferation, Senescence and Cell Death. Physiologia Plantarum, 119, 5-18. https://doi.org/10.1034/j.1399-3054.2003.00097.x

[104] Bi, X., Zhang, J., Chen, C., Zhang, D., Li, P. and Ma, F. (2014) Anthocyanin Contributes more to Hydrogen Peroxide Scavenging than Other Phenolics in Apple Peel. Food Chemistry, 152, 205-209. https://doi.org/10.1016/j.foodchem.2013.11.088

[105] Zhang, J., Chen, C., Zhang, D., Li, H., Li, P. and Ma, F. (2014) Reactive Oxygen Species Produced via Plasma Membrane NADPH Oxidase Regulate Anthocyanin Synthesis in Apple Peel. Planta, 240, 1023-1035. https://doi.org/10.1007/s00425-014-2120-4

[106] Łata, B., Trąmpczyńska, A. and Oleś, M. (2005) Antioxidant Content in the Fruit Peel, Flesh and Seeds of Selected Apple Cultivars during Cold Storage. Folia Horticulturae, 17, 47-60.

[107] Solovchenko, A. and Schmitz-Eiberger, M. (2003) Significance of Skin Flavonoids for UV-B-Protection in Apple Fruits. Journal of Experimental Botany, 54, 1977-1984. https://doi.org/10.1093/jxb/erg199 